\title{
AVALIAÇÃO REGIONAL DE CULTIVARES DE SOJA NO ESTADO DE SÃO PAULO - SAFRA 2018/19
}

\author{
BORGES, Wander Luis Barbosa ${ }^{1}$; HIPÓLITO, Jorge Luiz ${ }^{2}$; TOKUDA, Flávio Sueo ${ }^{3}$; MATEUS, \\ Gustavo Pavan ${ }^{4}$; BÁRBARO-TORNELI, Ivana Marino ${ }^{5}$; FINOTO, Everton Luis ${ }^{6}$; UNÊDA- \\ TREVISOLI, Sandra Helena ${ }^{7}$; CAZENTINI FILHO, Gerson ${ }^{8}$; FREITAS, Rogério Soares de ${ }^{1}$; \\ GASPARINO, Adriano Custódio ${ }^{9}$; LẼ̃O, Paulo César da Luz ${ }^{10}$
}

ISSUE DOI: $10.3738 / 1982.2278 .3625$

\begin{abstract}
RESUMO: A cada ano, as empresas de melhoramento criam novas cultivares para atender as demandas dos produtores, que tem diante de si, a necessidade cada vez maior de produzir mais, apesar das dificuldades. O presente trabalho teve por objetivo avaliar a adaptação de diferentes cultivares de soja, às condições edafoclimáticas do Estado de São Paulo. Os parâmetros avaliados na cultura da soja foram: altura de inserção da primeira vagem, altura de plantas, estande final ha ${ }^{-1}$, massa de mil grãos e produtividade de grãos. O delineamento experimental utilizado foi em blocos casualizados. Os dados foram submetidos ao teste $\mathrm{F}$ e as médias foram comparadas pelo teste de ScottKnott $(\mathrm{p}<0,05)$. Constatou-se que as cultivares avaliadas apresentaram, em média, massa de cem grãos variando de 12,37 a 17,19 g e produtividade de grãos variando de 2172 a $3471 \mathrm{~kg} \mathrm{ha}^{-1}$, nos oito locais avaliados no Estado de São Paulo.
\end{abstract}

Palavras-chave: Glycine max (L.) Merrill. Estabilidade de produção. Características agronômicas.

\section{SOYBEAN CULTIVARS REGIONAL EVALUATION IN SÃO PAULO STATE, BRAZIL - SEASON 2017/18}

\begin{abstract}
SUMMARY: Each year, breeding companies create new cultivars to meet the demands of producers, who have before them the growing need to produce more despite the difficulties. The present work had the objective of evaluating the adaptation of different soybean cultivars, to the edaphoclimatic conditions of São Paulo, State, Brazil. The parameters evaluated in the soybean crop were: height of insertion of the first pod, height of plants, final stand $\mathrm{ha}^{-1}$, mass of a thousand grains and grain yield. The experimental design was in randomized complete block design. The data were submitted to the F test and the means were compared by the Scott-Knott test ( $p<0.05)$. It was verified that the cultivars evaluated had, in average, one hundred grain mass varying from 12.37 to $17.19 \mathrm{~g}$ and grain productivity varying from 2172 to $3471 \mathrm{~kg} \mathrm{ha}^{-1}$, in the eight sites evaluated in São Paulo State.
\end{abstract}

Keywords: Glycine $\max ($ L.) Merrill. Production stability. Agronomic characteristics.

\section{INTRODUÇÃO}

Para uma planta manifestar o seu máximo potencial genético, caracterizado pelo seu melhor crescimento e desenvolvimento, diversos fatores ambientais podem influenciar diretamente no processo,

\footnotetext{
${ }^{1}$ Pesquisador Científico, Dr. - IAC - CAP Seringueira e Sistemas Agroflorestais, Votuporanga, SP;

${ }^{2}$ Assistente Agropecuário - CDRS - DSMM - NPS, Araçatuba, SP;

${ }^{3}$ Assistente Agropecuário - CDRS - DSMM - NPS, Fernandópolis, SP;

${ }^{4}$ Pesquisador Científico, Dr. - APTA - PRDTA Extremo Oeste, Andradina, SP;

${ }^{5}$ Pesquisadora Científica, Dra. - APTA - PRDTA da Alta Mogiana, Colina, SP;

${ }^{6}$ Pesquisador Científico, Dr. - APTA - PRDTA Centro Norte, Pindorama, SP;

${ }^{7}$ Professora Associada, Dra. - UNESP - FCAV, Jaboticabal, SP;

${ }^{8}$ Assistente Agropecuário - CDRS - DSMM - NPS, Manduri, SP;

${ }^{9}$ Assistente Agropecuário - CDRS - CA Américo de Campos, Américo de Campos, SP;

${ }^{10}$ Assistente Agropecuário - CDRS - CA Orlândia, Orlândia, SP.
} 
como fotoperíodo, temperatura, radiação solar, nutrientes e vento (YUYAMA, 1991).

A cada ano as empresas de melhoramento criam novas cultivares para atender as demandas dos produtores que tem diante de si a necessidade cada vez maior de produzir mais, apesar das dificuldades. Saber escolher a combinação de cultivares que irá resultar na melhor produtividade é tarefa importante e depende do conhecimento profundo dos fatores restritivos presentes na área a ser explorada. Além disso, o conhecimento das características das cultivares e de suas interações com os fatores de produção, formará a base de informações que irá determinar as melhores cultivares para cada área de cultivo (FUNDAÇÃO MT, 2007).

Por esse motivo, é desejável que os produtores tenham conhecimentos mais aprofundados das cultivares disponíveis em diferentes ambientes (KOMORI et al., 2004), pois, há grande variabilidade entre cultivares de soja com relação à sensibilidade à época e local de semeadura (PEIXOTO et al., 2000), visto que, em latitudes semelhantes ocorrem disponibilidade térmicas diferentes, tornando-se necessário a realização de ensaios de campo para se conhecer a fenologia das diferentes cultivares (VERNETTI, 1983) e, por as mais adaptadas, apresentarem maiores níveis de produtividade (YUYAMA, 1991).

Assim, as avaliações regionais são de suma importância, pois fomentam os sojicultores na escolha dos materiais mais adaptados às condições de clima e de solo de determinada região. O presente trabalho teve por objetivo avaliar a adaptação de diferentes cultivares de soja, às condições edafoclimáticas do Estado de São Paulo.

\section{MATERIAL E MÉTODO}

O experimento foi desenvolvido em Andradina, SP, no Polo Regional de Desenvolvimento Tecnológico dos Agronegócios (PRDTA) do Extremo Oeste, da Agência Paulista de Tecnologia dos Agronegócios (APTA), da Secretaria de Agricultura e Abastecimento do Estado de São Paulo - SAA; em Araçatuba, SP, na Fazenda Santo Antônio (propriedade particular); em Colina, SP, no PRDTA Alta Mogiana, da APTA, da SAA; em Jaboticabal, na Fazenda de Ensino, Pesquisa e Extensão da Faculdade de Ciências Agrárias e Veterinárias - FCAV, da Universidade Estadual Paulista e UNESP; em Manduri, SP, na Fazenda Ataliba Leonel, do Núcleo de Produção de Mudas e Sementes - NPMS, do Departamento de Sementes, Mudas e Matrizes - DSMM, da Coordenadoria de Desenvolvimento Rural Sustentável (CDRS), da SAA; em Pindorama, SP, no PRDTA do Centro Norte, da APTA, da SAA; em Riolândia, SP, na Fazenda Compasso (propriedade particular); em Votuporanga, SP, no Centro Avançado de Pesquisa de Seringueira e Sistemas Agroflorestais, do Instituto Agronômico - IAC, da APTA, da SAA.

Em Andradina, Araçatuba, Jaboticabal, Pindorama, Riolândia e Votuporanga o clima é o tropical com invernos secos (Aw na classificação de Köppen), com temperatura média anual de $24^{\circ} \mathrm{C}$, tendo a média das máximas de $31,2{ }^{\circ} \mathrm{C}$ e a média das mínimas de $17,4^{\circ} \mathrm{C}$. A precipitação pluviométrica média anual é de 1328,6 mm.

Em Colina o clima é o tropical de altitude com inverno seco (Cwa na classificação de Köppen), ou seja, com temperatura média anual de $22,2^{\circ} \mathrm{C}$ e precipitação média anual de $1451 \mathrm{~mm}$.

Em Manduri, o clima é quente e temperado (Cfa na classificação de Köppen), com temperatura média anual de $19,8^{\circ} \mathrm{C}$ e precipitação média anual de $1249 \mathrm{~mm}$.

O delineamento experimental utilizado foi em blocos casualizados com 32 tratamentos (cultivares de soja) e 4 repetições em Andradina, Araçatuba, Pindorama, Riolândia e Votuporanga; 34 tratamentos e 3 repetições em Colina e Jaboticabal; 35 tratamentos e 4 repetições em Manduri. 
Devido a problemas com germinação de sementes, o que refletiu em um estande final de plantas inadequado, na análise estatística dos dados de Manduri foram utilizadas somente 32 cultivares, na de Colina 28 e na de Votuporanga 24.

Amostras de solo para caracterização química (RAIJ et al., 2001) e granulométrica (DAY, 1965) foram coletadas em outubro de 2017, na camada de 0-0,20 m de profundidade, e os resultados estão apresentados na Tabela 1.

Tabela 1. Caracterização química e granulométrica do solo, na camada de 0-0,20 m, 2018.

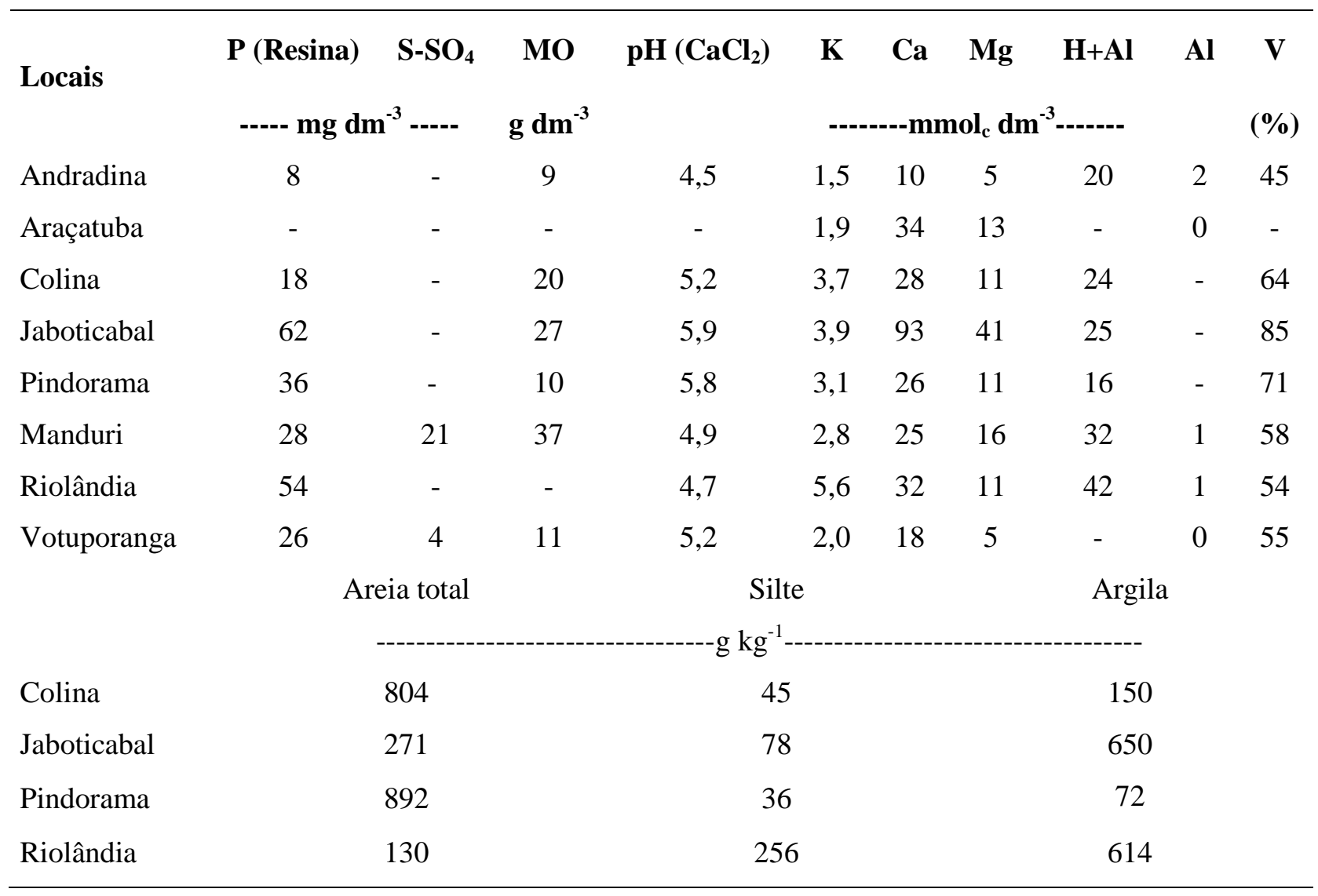

Em outubro de 2018, realizada uma calagem com calcário dolomítico na dose de $1100 \mathrm{~kg} \mathrm{ha}^{-1}$, em Andradina e uma calagem superficial com calcário calcítico, na dose de $1000 \mathrm{~kg} \mathrm{ha}^{-1}$, em Araçatuba.

As parcelas experimentais foram compostas por quatro linhas de $5 \mathrm{~m}$, com espaçamento de $0,5 \mathrm{~m}$ entre linhas, utilizando-se as duas linhas centrais para as avaliações agronômicas.

Os tratamentos constaram dos seguintes obtentores ou multiplicadores e suas respectivas cultivares:

- Agroeste: AS 3610 IPRO, AS 3590 IPRO, AS 3680 IPRO, AS 3730 IPRO;

- Caraíba Genética e Sementes: Caraíba Speed, Caraíba Nitro;

- GDM Genética: 64I61RSF (Brasmax Fibra IPRO), DM 66i68 RSF IPRO, 68i70 RSF (Brasmax Ícone IPRO), $68 \mathrm{I} 68$ RSF (Brasmax Única IPRO), DM $68 \mathrm{i} 69$ IPRO, 74I77 RSF (Brasmax Foco IPRO);

- Seedcorp/HO: HO Aporé IPRO, HO Corumbá IPRO, HO Cristalino IPRO, HO Iguaçu IPRO, HO Maracaí IPRO;

- LG Sementes: LG 60158 IPRO, LG 60162 IPRO, LG 60174 IPRO, LG 60179 IPRO;

- Nidera Sementes: NS 6700 IPRO, NS 6906 IPRO, NS 7667 IPRO, NS 7709 IPRO;

- Bela Sementes: TEC 6702 IPRO, TMG 7067 IPRO; 
- Embrapa: BRS 284, BRS 511, BRS Valiosa RR;

- Instituto Agronômico - IAC: IAC Foscarin-31.

Também foram utilizadas 6 cultivares codificadas: 1 XT-I, 2 XN, 3 YM-I, 4 YB-I, 5 KW e 6 WBI.

A semeadura foi realizada nos dias 12/11/2018 em Andradina; 12 e 13/11/2018 em Araçatuba; 06/11/2018 em Colina; 13/11/2018 em Jaboticabal; 05/12/2018 em Pindorama; 09/11/2018 em Manduri, 21/11/2018 em Riolândia e 12/11/2018 em Votuporanga. Utilizou-se semeadora de parcelas e/ou carriolas semeadoras, com preparo convencional do solo em Andradina, Araçatuba, Pindorama, Riolândia e Votuporanga e, sistema de semeadura direta, sobre palhada de crotalária em Colina e de braquiária em Jaboticabal e em Manduri.

$\mathrm{Na}$ adubação de semeadura foi utilizado $250 \mathrm{~kg} \mathrm{ha}^{-1}$ do adubo formulado 04-30-10 em Andradina; $220 \mathrm{~kg} \mathrm{ha}^{-1}$ do adubo formulado 08-52-00 em Araçatuba; $300 \mathrm{~kg} \mathrm{ha}^{-1}$ do adubo formulado 04-20-20 em Colina; $300 \mathrm{~kg} \mathrm{ha}^{-1}$ do adubo formulado 02-20-20 com 8\% de Ca e 4\% de S em Jaboticabal; $350 \mathrm{~kg} \mathrm{ha}^{-1}$ do adubo formulado 04-30-16 em Pindorama; $300 \mathrm{~kg} \mathrm{ha}^{-1}$ do adubo formulado 08-24-12 em Manduri; $250 \mathrm{~kg} \mathrm{ha}^{-1}$ do adubo formulado 05-25-00 em Riolândia; $150 \mathrm{~kg} \mathrm{ha}^{-1}$ do adubo formulado 08-28-16 em Votuporanga.

Em Araçatuba, Manduri e Riolândia, foi realizada uma adubação com cloreto de potássio aos 25 dias após a germinação, na dose de 150, 100 e $60 \mathrm{~kg} \mathrm{ha}^{-1}$, respectivamente.

A adubação de semeadura foi feita mecanicamente com semeadoras de grãos. Quinze dias após a semeadura foi realizado o desbaste de plantas, deixando-se a população inicial recomendada pelos detentores de cada cultivar.

Os parâmetros avaliados foram: altura de inserção da primeira vagem e altura de plantas (em metro), estande final $\mathrm{ha}^{-1}$, massa de cem grãos (em gramas) e produtividade de grãos (em kg ha ${ }^{-1}$ ). Em Colina e Jaboticabal também avaliou-se o índice de acamamento, de acordo com escala de notas proposta por Bernard et al. (1965), em que se atribuiu nota de 1,0 (todas as plantas eretas) a 5,0 (todas as plantas acamadas); o valor agronômico da planta, por meio de uma escala de notas visuais, a qual varia de 1,0 (plantas com características agronômicas ruins) a 5,0 (plantas com ótimas características agronômicas), sendo a nota atribuída representativa de um conjunto de caracteres visuais (arquitetura da planta, quantidade de vagens cheias, vigor e sanidade da planta, debulha prematura das vagens, acamamento e retenção foliar na maturidade).

Em Jaboticabal atribuiu-se para o estande final uma escala de 1,0 (estande muito inadequado, com muitas falhas na parcela) a 5,0 (estande muito bom, sem falhas na parcela). Também mensurou-se o índice de acamamento em Riolândia, no entanto, todas as plantas receberam nota 1 (todas as plantas eretas) e por isso não foi realizada a análise estatística para essa característica, neste local. Em Colina, Jaboticabal, Pindorama e Riolândia, verificou-se o número de dias para a maturação. Em Votuporanga anotou-se o ciclo das cultivares (em dias) do momento da semeadura à colheita e, em Pindorama, o número de dias até o pleno florescimento.

As avaliações foram realizadas no momento da colheita da soja, realizada nos dias 29/03/2019 em Colina, 25/03/2019 em Jaboticabal, 04/04/2018 em Pindorama. Em Andradina a colheita foi realizada no período de 07/03/2019 a 28/03/2019, em Araçatuba de 10/02/2019 a 15/03/2019, em Riolândia de 27/02/2019 a 18/03/2019, em Votuporanga, de 05/03/2019 a 01/04/2019, em Manduri na segunda quinzena de março de 2019.

A amostragem da altura de inserção da primeira vagem e altura de plantas foi realizada em cinco plantas aleatórias de cada parcela, e a amostragem do estande final $\mathrm{ha}^{-1}$, massa de cem grãos e produtividade de grãos foi realizada em $5 \mathrm{~m}$ das duas linhas centrais de cada parcela em Manduri e 
Riolândia; em 3 m das duas linhas centrais de cada parcela em Andradina, Colina, Pindorama e Votuporanga; em $2 \mathrm{~m}$ das duas linhas centrais em Araçatuba.

Em Jaboticabal utilizou-se uma colhedora de parcelas, amostrando-se $4 \mathrm{~m}^{2}$ por parcela, para análise da massa de cem grãos e produtividade de grãos. Nos demais locais as vagens foram debulhadas em debulhadora mecânica. Após a debulha os grãos foram pesados e mensurada sua umidade para o cálculo da produtividade de grãos. Em seguida separou-se cem grãos para obtenção da massa de cem grãos. A massa de cem grãos e a produtividade de grãos foi obtida padronizando-se a umidade dos grãos para $13 \%$ (base úmida).

Realizou-se a análise conjunta dos valores médios da altura de inserção da primeira vagem, altura de plantas, estande final ha ${ }^{-1}$, massa de mil grãos e produtividade de grãos, de 32 cultivares que foram utilizadas nos oito locais.

A resenha agrometeorológica de Andradina, Araçatuba, Colina, Ribeirão Preto (Estação meteorológica mais próxima à Jaboticabal), Manduri, Pindorama, Cardoso (Estação meteorológica mais próxima à Riolândia) e Votuporanga encontram-se nas Figuras 1, 2 e 3.

Figura 1. Valores médios mensais de temperatura média mínima e máxima em cada local de instalação, no período de 01/11/2018 a 01/04/2018. Fonte: CIIAGRO (2019).

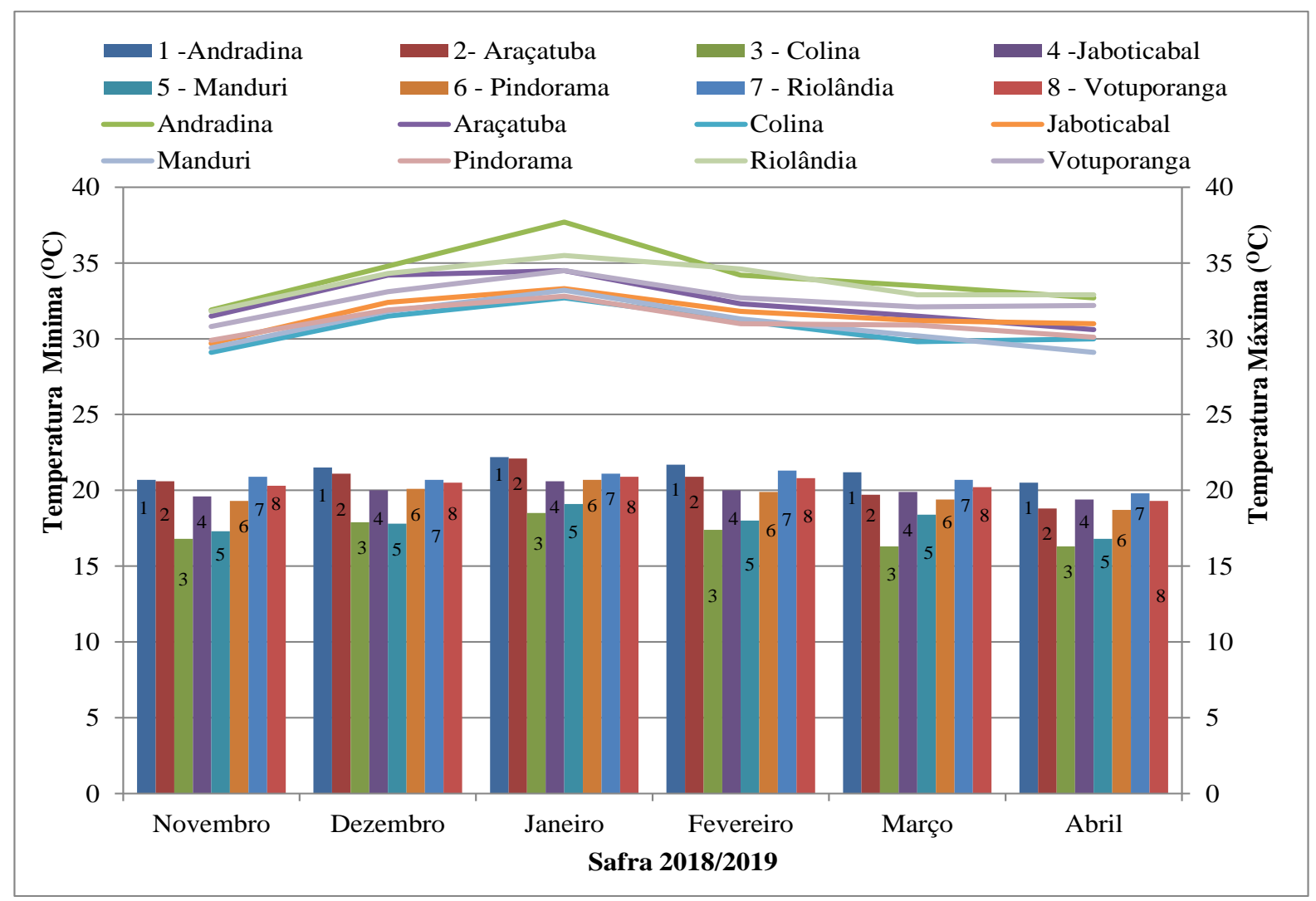


Figura 2. Valores médios mensais de evapotranspiração cada local de instalação do ensaio, no período de 01/11/2018 a 01/04/2018. Fonte: CIIAGRO (2019).

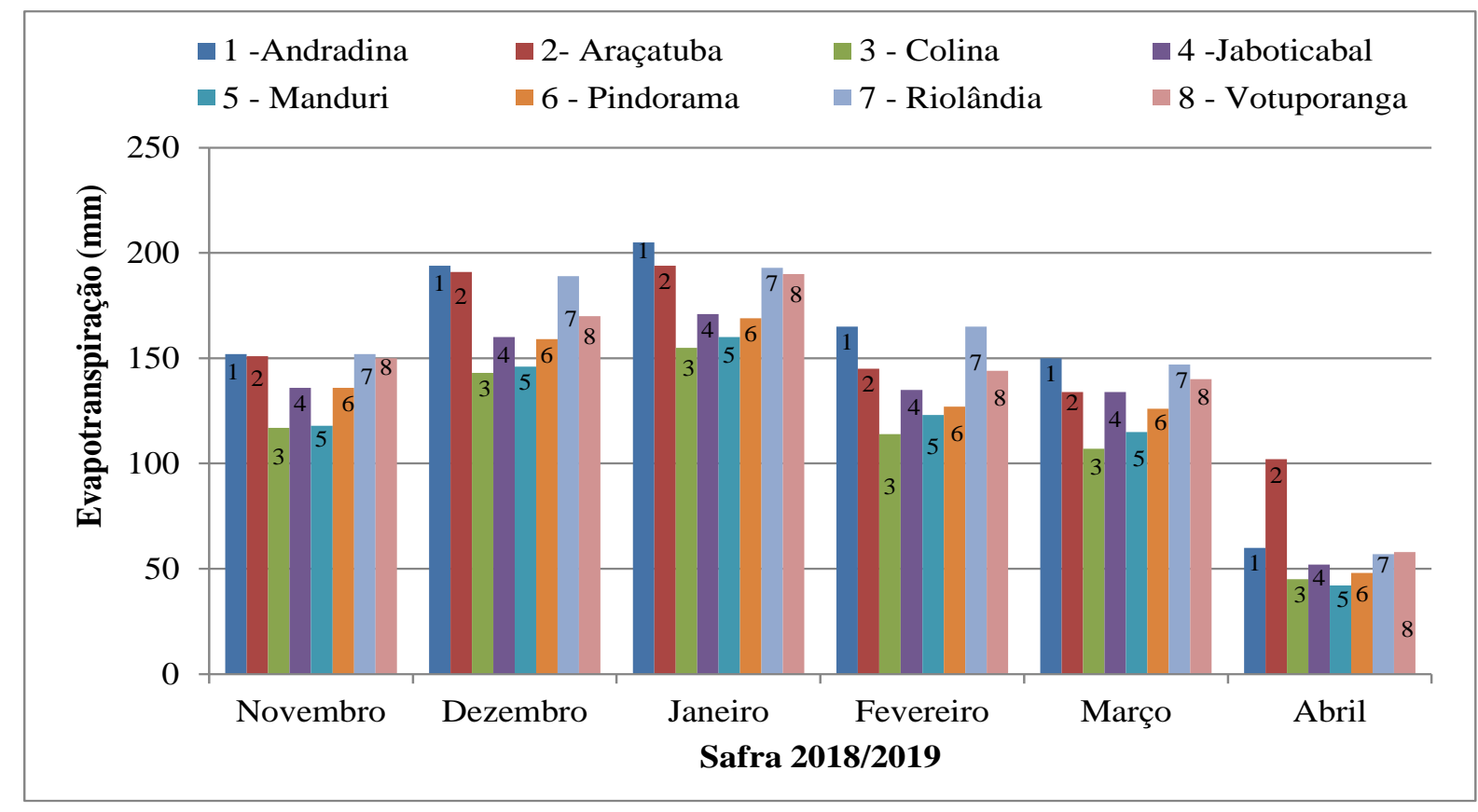

Figura 3. Valores médios mensais de precipitação cada local de instalação do ensaio no período de 01/11/2018 a 01/04/2018. Fonte: CIIAGRO (2019).

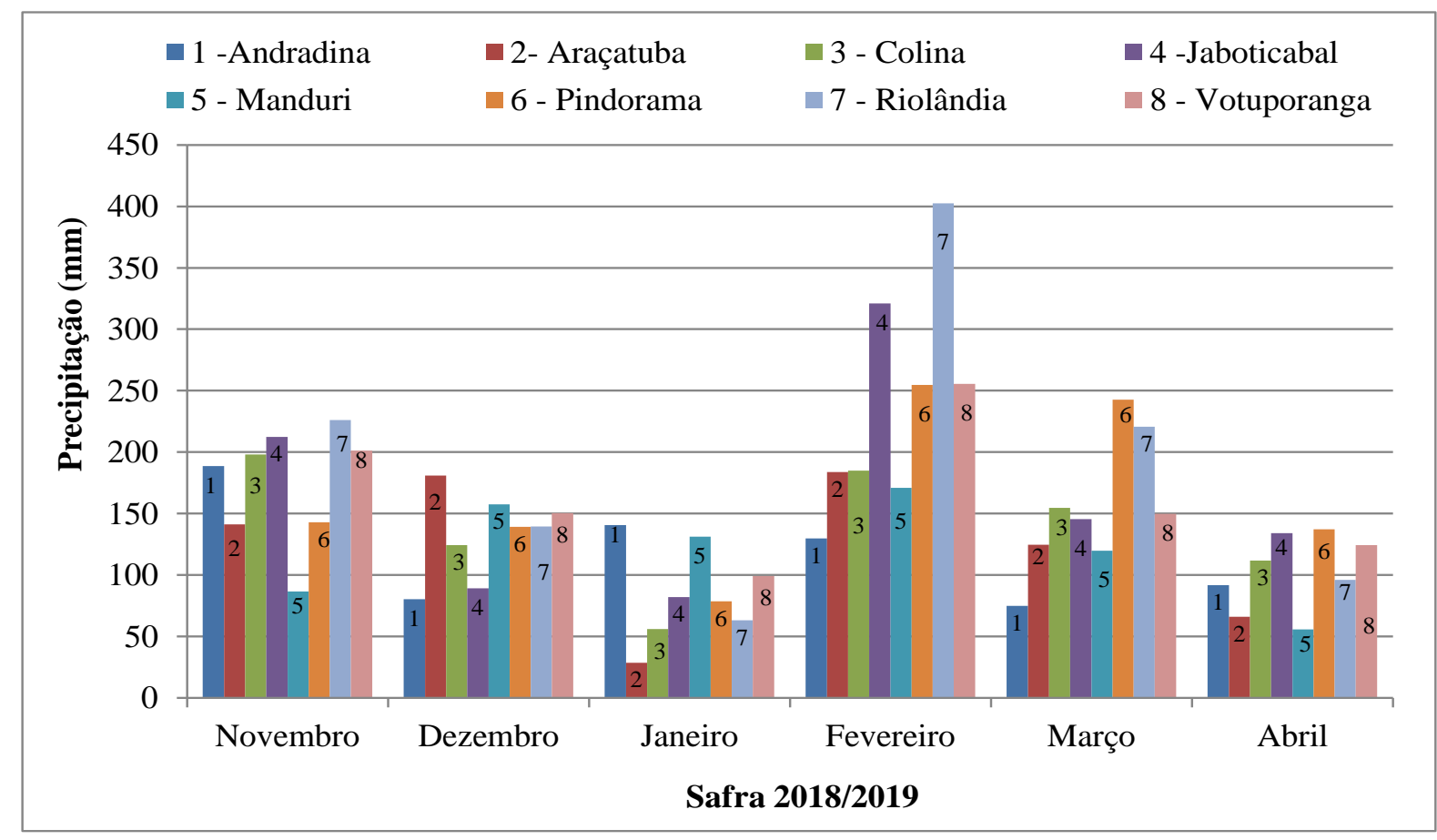

Os dados foram submetidos ao teste $\mathrm{F}$ e as médias foram comparadas pelo teste de Scott-Knott ( $\mathrm{p}<0,05$ ), com o uso do programa computacional Assistat (SILVA; AZEVEDO, 2016).

Não se comparou as médias dos estandes finais, números de dias para a maturação, número de dias até o pleno florescimento e ciclos das cultivares, por variarem de acordo com a recomendação do detentor de cada cultivar. 


\section{RESULTADO E DISCUSSÃO}

As características agronômicas da cultura da soja, nos oitos locais, estão demonstradas nas Tabelas 02 a 12. Destaca-se a variabilidade das cultivares entre os locais. Segundo Yuyama (1991), o sucesso em relação à produtividade da cultivar é condicionado pelo genótipo do material e pela sua interação com as variações ambientais.

Na Tabela 02 estão demonstradas as características agronômicas da cultura da soja de Andradina. As diferentes cultivares diferiram entre si $(\mathrm{p}<0,05)$ em relação à altura de inserção da primeira vagem, altura de plantas e massa de cem grãos,

Tabela 02. Características agronômicas avaliadas em diferentes cultivares de soja, Andradina, SP, 2019.

(Continua)

\begin{tabular}{|c|c|c|c|c|c|}
\hline Cultivar & $\begin{array}{l}\text { Altura de } \\
\text { inserção }^{(6)}\end{array}$ & $\begin{array}{l}\text { Altura de } \\
\text { plantas }\end{array}$ & $\begin{array}{c}\text { Estande final } \\
\text { (ha) }\end{array}$ & $\begin{array}{c}\text { Massa de } \\
\text { cem grãos } \\
\text { (g) }\end{array}$ & $\begin{array}{c}\text { Produtividade } \\
\text { de grãos } \\
\left(\mathrm{kg} \mathrm{ha}^{-1}\right)\end{array}$ \\
\hline AS 3610 IPRO & $0,16 d^{(7)}$ & $0,54 \mathrm{c}$ & 318515 & $14,85 \mathrm{c}$ & 1950 \\
\hline AS 3590 IPRO & $0,17 \mathrm{~d}$ & $0,59 \mathrm{c}$ & 259257 & $14,70 \mathrm{c}$ & 2184 \\
\hline AS 3680 IPRO & $0,15 \mathrm{~d}$ & $0,62 \mathrm{c}$ & 279627 & $12,64 \mathrm{~d}$ & 2032 \\
\hline Caraíba Speed & $0,12 \mathrm{e}$ & $0,55 \mathrm{c}$ & 347219 & $12,35 \mathrm{~d}$ & 2361 \\
\hline Caraíba Nitro & $0,12 \mathrm{e}$ & $0,52 \mathrm{~d}$ & 320367 & $13,61 \mathrm{~d}$ & 1768 \\
\hline 64i61RSF ${ }^{(1)}$ & $0,14 \mathrm{e}$ & $0,61 \mathrm{c}$ & 305553 & $13,10 \mathrm{~d}$ & 2163 \\
\hline DM $66 i 68$ RSF IPRO & 0,14 e & $0,57 \mathrm{c}$ & 305553 & $17,34 \mathrm{~b}$ & 2227 \\
\hline $68 \mathrm{i} 70 \mathrm{RSF}^{(2)}$ & $0,15 \mathrm{~d}$ & $0,56 \mathrm{c}$ & 282405 & $17,75 \mathrm{~b}$ & 2807 \\
\hline $68 \mathrm{i} 68 \mathrm{RSF}^{(3)}$ & $0,12 \mathrm{e}$ & $0,56 \mathrm{c}$ & 306478 & $16,87 \mathrm{~b}$ & 2353 \\
\hline DM $68 \mathrm{i} 69$ IPRO & 0,14 e & $0,55 \mathrm{c}$ & 290738 & $14,31 \mathrm{c}$ & 2539 \\
\hline $74 \mathrm{i} 77 \mathrm{RSF}^{(4)}$ & $0,11 \mathrm{e}$ & $0,59 \mathrm{c}$ & 235183 & $14,99 \mathrm{c}$ & 2091 \\
\hline HO Aporé IPRO & $0,12 \mathrm{e}$ & $0,59 \mathrm{c}$ & 149073 & $14,43 \mathrm{c}$ & 2348 \\
\hline HO Corumbá IPRO & 0,13 e & $0,56 \mathrm{c}$ & 296293 & $16,21 \mathrm{~b}$ & 2298 \\
\hline HO Cristalino IPRO & $0,24 \mathrm{~b}$ & 0,78 a & 295367 & $15,74 \mathrm{c}$ & 2888 \\
\hline HO Maracaí IPRO & 0,27 a & 0,74 a & 278701 & 18,65 a & 2229 \\
\hline HO Iguaçu IPRO & $0,15 \mathrm{~d}$ & $0,59 \mathrm{c}$ & 362033 & $13,08 \mathrm{~d}$ & 2169 \\
\hline LG 60158 IPRO & $0,12 \mathrm{e}$ & $0,47 \mathrm{~d}$ & 308330 & $14,37 \mathrm{c}$ & 2231 \\
\hline LG 60162 IPRO & $0,13 \mathrm{e}$ & $0,48 \mathrm{~d}$ & 279627 & $13,04 \mathrm{~d}$ & 2119 \\
\hline LG 60174 IPRO & $0,12 \mathrm{e}$ & $0,63 \mathrm{c}$ & 258331 & $14,34 \mathrm{c}$ & 2383 \\
\hline LG 60179 IPRO & $0,20 \mathrm{c}$ & 0,80 a & 316664 & $17,23 \mathrm{~b}$ & 3459 \\
\hline NS 6906 IPRO & $0,14 \mathrm{~d}$ & $0,54 \mathrm{c}$ & 274997 & $15,62 \mathrm{c}$ & 2202 \\
\hline NS 7709 IPRO & $0,17 \mathrm{~d}$ & $0,65 \mathrm{~b}$ & 295367 & $18,02 \mathrm{~b}$ & 2513 \\
\hline NS 7667 IPRO & $0,17 \mathrm{~d}$ & $0,66 \mathrm{~b}$ & 240738 & $17,71 \quad b$ & 2247 \\
\hline
\end{tabular}


Tabela 02. Características agronômicas avaliadas em diferentes cultivares de soja, Andradina, SP, 2019.

(Conclusão)

\begin{tabular}{|c|c|c|c|c|c|}
\hline Cultivar & $\begin{array}{l}\text { Altura de } \\
\text { inserção }^{(6)}\end{array}$ & $\begin{array}{l}\text { Altura de } \\
\text { plantas }\end{array}$ & $\begin{array}{c}\text { Estande final } \\
\text { (ha) }\end{array}$ & $\begin{array}{c}\text { Massa de } \\
\text { cem grãos } \\
(\mathrm{g})\end{array}$ & $\begin{array}{l}\text { Produtividade } \\
\text { de grãos } \\
\left(\mathrm{kg} \mathrm{ha}^{-1}\right)\end{array}$ \\
\hline NS 6700 IPRO & $0,23 \mathrm{~b}$ & $0,70 \mathrm{~b}$ & 281479 & 19,83 a & 2587 \\
\hline TEC 6702 IPRO & $0,16 \mathrm{~d}$ & $0,60 \mathrm{c}$ & 244442 & 18,66 a & 2454 \\
\hline TMG 7067 IPRO & $0,16 \mathrm{~d}$ & $0,57 \mathrm{c}$ & 308330 & $11,55 \mathrm{~d}$ & 1553 \\
\hline $1 \mathrm{XT}-\mathrm{I}$ & $0,17 \mathrm{~d}$ & $0,58 \mathrm{c}$ & 235183 & $14,64 \mathrm{c}$ & 1925 \\
\hline $2 \mathrm{XN}$ & $0,10 \mathrm{e}$ & $0,54 \mathrm{c}$ & 128702 & $15,34 \mathrm{c}$ & 2039 \\
\hline 3 YM-I & $0,18 \mathrm{~d}$ & $0,44 \mathrm{~d}$ & 298145 & $12,63 \mathrm{~d}$ & 1933 \\
\hline 4 YB-I & $0,12 \mathrm{e}$ & $0,52 \mathrm{~d}$ & 310182 & $14,80 \mathrm{c}$ & 2440 \\
\hline $5 \mathrm{KW}$ & $0,15 \mathrm{~d}$ & $0,57 \mathrm{c}$ & 242590 & $13,58 \mathrm{~d}$ & 1836 \\
\hline 6 WB-I & $0,15 \mathrm{~d}$ & $0,55 \mathrm{c}$ & 288886 & $14,10 \mathrm{c}$ & 2025 \\
\hline $\mathrm{CV}^{(5)}(\%)$ & 14,33 & 10,12 & & 8,407 & 21,47 \\
\hline
\end{tabular}

Não houve diferença entre as cultivares $(\mathrm{p}<0,05)$ em relação à produtividade de grãos, sendo que a produtividade média foi de $2261 \mathrm{~kg} \mathrm{ha}^{-1}$. Destaca-se que nos meses de dezembro, janeiro e fevereiro houve acentuada evapotranspiração no local, com média de temperaturas máximas acima de $38^{\circ} \mathrm{C}$ e temperaturas médias acima de $28^{\circ} \mathrm{C}$ (Figuras 1 e 2), o que prejudicou o desenvolvimento vegetativo e reprodutivo da cultura.

As características agronômicas da cultura da soja, em Araçatuba, estão demonstradas na Tabela 03. As diferentes cultivares diferiram entre si $(\mathrm{p}<0,05)$ em relação à altura de inserção da primeira vagem, altura de plantas, massa de cem grãos e produtividade de grãos, corroborando com Cruz et al. (2010), que também encontraram diferenças significativas entre as cultivares para as variáveis massa de mil grãos e produtividade de grãos, e enfatizaram que isso mostra que os genótipos apresentaram características agronômicas bastante diferenciadas.

Tabela 03. Características agronômicas avaliadas em diferentes cultivares de soja, Araçatuba, SP, 2019.

(Continua)

\begin{tabular}{lccccc}
\hline Cultivar & $\begin{array}{c}\text { Altura de } \\
\text { inserção }^{(\mathbf{6})}\end{array}$ & $\begin{array}{c}\text { Altura de } \\
\text { plantas }\end{array}$ & $\begin{array}{c}\text { Estande final } \\
(\mathbf{m})\end{array}$ & $\begin{array}{c}\text { Massa de } \\
\text { cem grãos }\end{array}$ & $\begin{array}{c}\text { Produtividade } \\
\text { de grãos }\end{array}$ \\
AS 3610 IPRO & $0,14 \mathrm{c}^{(7)}$ & $0,66 \mathrm{c}$ & 192500 & $\begin{array}{c}(\mathbf{g}) \\
\mathbf{( k g ~ h a}^{-1} \mathbf{)}\end{array}$ \\
AS 3590 IPRO & $0,14 \mathrm{c}$ & $0,61 \mathrm{c}$ & 204875 & $11,09 \mathrm{~d}$ & $1546 \mathrm{~b}$ \\
AS 3680 IPRO & $0,14 \mathrm{c}$ & $0,74 \mathrm{~b}$ & 188375 & $13,12 \mathrm{~b}$ & $1900 \mathrm{a}$ \\
Caraíba Speed & $0,13 \mathrm{c}$ & $0,62 \mathrm{c}$ & 220000 & $10,89 \mathrm{~d}$ & $2213 \mathrm{a}$ \\
Caraíba Nitro & $0,13 \mathrm{c}$ & $0,60 \mathrm{c}$ & 222750 & $11,87 \mathrm{c}$ & $2020 \mathrm{a}$ \\
\hline
\end{tabular}

Nucleus, Edição Especial, 2019 
Tabela 03. Características agronômicas avaliadas em diferentes cultivares de soja, Araçatuba, SP, 2019.

(Conclusão)

\begin{tabular}{|c|c|c|c|c|c|}
\hline Cultivar & $\begin{array}{l}\text { Altura de } \\
\text { inserção }^{(6)}\end{array}$ & $\begin{array}{l}\text { Altura de } \\
\text { plantas }\end{array}$ & $\begin{array}{c}\text { Estande final } \\
\text { (ha) }\end{array}$ & $\begin{array}{c}\text { Massa de } \\
\text { cem grãos } \\
\text { (g) }\end{array}$ & $\begin{array}{c}\text { Produtividade } \\
\text { de grãos } \\
\left(\mathrm{kg} \mathrm{ha}^{-1}\right)\end{array}$ \\
\hline $64 \mathrm{i} 61 \mathrm{RSF}^{(1)}$ & $0,15 \mathrm{c}$ & 0,76 a & 243375 & $9,40 \mathrm{e}$ & 1919 a \\
\hline DM 66i68 RSF IPRO & $0,15 \mathrm{c}$ & $0,66 \mathrm{c}$ & 226875 & $13,08 \mathrm{~b}$ & $1683 \mathrm{~b}$ \\
\hline $68 \mathrm{i} 70 \mathrm{RSF}^{(2)}$ & $0,14 \mathrm{c}$ & $0,63 \mathrm{c}$ & 251625 & $12,01 \mathrm{c}$ & $1725 \mathrm{~b}$ \\
\hline $68 \mathrm{i} 68 \mathrm{RSF}^{(3)}$ & $0,15 \mathrm{c}$ & $0,71 \mathrm{~b}$ & 239250 & $13,13 \mathrm{~b}$ & 2149 a \\
\hline DM 68169 IPRO & $0,15 \mathrm{c}$ & $0,66 \mathrm{c}$ & 217250 & $12,45 \mathrm{c}$ & $1655 \mathrm{~b}$ \\
\hline 74i77 $\mathrm{RSF}^{(4)}$ & $0,16 \mathrm{c}$ & 0,75 a & 195250 & 9,62 e & 2191 a \\
\hline HO Aporé IPRO & $0,14 \mathrm{c}$ & $0,70 \mathrm{~b}$ & 202125 & $11,92 \mathrm{c}$ & $1768 \mathrm{~b}$ \\
\hline HO Corumbá IPRO & $0,15 \mathrm{c}$ & $0,74 \mathrm{~b}$ & 232375 & $11,87 \mathrm{c}$ & 1934 a \\
\hline HO Cristalino IPRO & $0,26 \mathrm{~b}$ & 0,80 a & 207625 & $13,58 \mathrm{~b}$ & 2188 a \\
\hline HO Maracaí IPRO & $0,28 \mathrm{~b}$ & 0,81 a & 176000 & $12,87 \mathrm{~b}$ & 1894 a \\
\hline HO Iguaçu IPRO & $0,18 \mathrm{c}$ & $0,69 \mathrm{~b}$ & 237875 & $10,02 \mathrm{e}$ & 2040 a \\
\hline LG 60158 IPRO & $0,12 \mathrm{c}$ & $0,52 \mathrm{~d}$ & 217250 & $11,07 \mathrm{~d}$ & 1892 a \\
\hline LG 60162 IPRO & $0,14 \mathrm{c}$ & $0,58 \mathrm{~d}$ & 207625 & $10,00 \mathrm{e}$ & 1492 b \\
\hline LG 60174 IPRO & $0,15 \mathrm{c}$ & $0,68 \mathrm{c}$ & 185625 & $10,79 \mathrm{~d}$ & 1976 a \\
\hline LG 60179 IPRO & $0,21 \mathrm{c}$ & 0,77 a & 180125 & $10,74 \mathrm{~d}$ & 2308 a \\
\hline NS 6906 IPRO & $0,14 \mathrm{c}$ & $0,68 \mathrm{~b}$ & 200750 & $11,65 \mathrm{~d}$ & 1986 a \\
\hline NS 7709 IPRO & $0,17 \mathrm{c}$ & $0,72 \mathrm{~b}$ & 185625 & $11,42 \mathrm{~d}$ & $1760 \mathrm{~b}$ \\
\hline NS 7667 IPRO & $0,19 \mathrm{c}$ & $0,69 \mathrm{~b}$ & 191125 & $11,37 \mathrm{~d}$ & 2073 a \\
\hline NS 6700 IPRO & $0,20 \mathrm{c}$ & $0,73 \mathrm{~b}$ & 228250 & $12,81 \mathrm{~b}$ & $1474 \mathrm{~b}$ \\
\hline TEC 6702 IPRO & 0,39 a & $0,65 \mathrm{c}$ & 210375 & 15,68 a & 1983 a \\
\hline TMG 7067 IPRO & $0,16 \mathrm{c}$ & $0,72 \mathrm{~b}$ & 185625 & $11,33 \mathrm{~d}$ & $1721 \mathrm{~b}$ \\
\hline $1 \mathrm{XT}-\mathrm{I}$ & $0,18 \mathrm{c}$ & $0,74 \mathrm{~b}$ & 233750 & $12,08 \mathrm{c}$ & 1978 a \\
\hline $2 \mathrm{XN}$ & $0,13 \mathrm{c}$ & $0,66 \mathrm{c}$ & 199375 & $11,27 \mathrm{~d}$ & 1934 a \\
\hline 3 YM-I & $0,19 \mathrm{c}$ & $0,65 \mathrm{c}$ & 177375 & $9,09 \mathrm{e}$ & 1901 a \\
\hline 4 YB-I & $0,13 \mathrm{c}$ & $0,61 \mathrm{c}$ & 221375 & $12,25 \mathrm{c}$ & 1924 a \\
\hline $5 \mathrm{KW}$ & $0,17 \mathrm{c}$ & $0,70 \mathrm{~b}$ & 202125 & $10,76 \mathrm{~d}$ & $1458 \mathrm{~b}$ \\
\hline $6 \mathrm{WB}-\mathrm{I}$ & $0,17 \mathrm{c}$ & 0,76 a & 193875 & $13,08 \mathrm{~b}$ & $1785 \mathrm{~b}$ \\
\hline $\mathrm{CV}^{(5)}(\%)$ & 31,37 & 7,53 & & 3,82 & 18,30 \\
\hline
\end{tabular}

\footnotetext{
${ }^{(1)}$ Brasmax Fibra IPRO; ${ }^{(2)}$ Brasmax Ícone IPRO; ${ }^{(3)}$ Brasmax Única IPRO; ${ }^{(4)}$ Brasmax Foco IPRO;

Coeficiente de variação; ${ }^{(6)}$ Altura de inserção: altura de inserção da primeira espiga; ${ }^{(7)}$ significativo a $5 \%$ de probabilidade.
}

A produtividade média de grãos foi de $1895 \mathrm{~kg} \mathrm{ha}^{-1}$. Destaca-se que a evapotranspiração foi bem acentuada nos meses de novembro, dezembro e, principalmente, em janeiro, fase de enchimento de grãos, no qual se registrou uma evapotranspiração de $194 \mathrm{~mm}$ e uma precipitação de apenas 28,6 mm e, nos três 
meses, temperaturas máximas acima de $36^{\circ} \mathrm{C}$ e temperaturas médias acima de $26^{\circ} \mathrm{C}$ (Figura 2), prejudicando o desenvolvimento vegetativo e reprodutivo da cultura.

Nas Tabelas 04 e 05 estão demonstradas as características agronômicas da cultura da soja de Colina. As diferentes cultivares diferiram entre si $(p<0,05)$ em relação à altura de inserção da primeira vagem, altura de plantas, massa de cem grãos e produtividade de grãos.

Tabela 04. Características agronômicas avaliadas em diferentes cultivares de soja, Colina, SP, 2019.

\begin{tabular}{|c|c|c|c|c|c|}
\hline Cultivar & $\begin{array}{c}\text { Altura de } \\
\text { inserção }^{(3)}\end{array}$ & $\begin{array}{l}\text { Altura de } \\
\text { plantas }\end{array}$ & $\begin{array}{c}\text { Estande } \\
\text { final } \\
\text { (ha) }\end{array}$ & $\begin{array}{l}\text { Massa de } \\
\text { cem grãos } \\
\text { (g) }\end{array}$ & $\begin{array}{c}\text { Produtividade de } \\
\text { grãos } \\
\left(\mathrm{kg} \mathrm{ha}^{-1}\right)\end{array}$ \\
\hline AS 3610 IPRO & $0,12 b^{(4)}$ & $0,80 \mathrm{c}$ & 275333 & $12,83 \mathrm{~d}$ & $1104 \mathrm{i}$ \\
\hline AS 3590 IPRO & 0,16 a & $0,77 \mathrm{~d}$ & 228667 & $14,40 \mathrm{c}$ & $1948 \mathrm{f}$ \\
\hline AS 3680 IPRO & 0,16 a & $0,85 \mathrm{c}$ & 381333 & $14,17 \mathrm{c}$ & $2016 \mathrm{e}$ \\
\hline AS 3730 IPRO & $0,10 \mathrm{c}$ & $0,76 \mathrm{~d}$ & 201333 & $14,40 \mathrm{c}$ & $1849 \mathrm{f}$ \\
\hline Caraíba Speed & 0,15 a & $0,67 \mathrm{e}$ & 322000 & $13,97 \mathrm{c}$ & 2048 e \\
\hline Caraíba Nitro & $0,11 \mathrm{~b}$ & $0,66 \mathrm{e}$ & 231333 & $15,53 \mathrm{~b}$ & $1854 \mathrm{f}$ \\
\hline $64 \mathrm{i} 61 \mathrm{RSF}^{(1)}$ & $0,13 \mathrm{~b}$ & $0,78 \mathrm{~d}$ & 264667 & $13,00 \mathrm{~d}$ & $2161 \mathrm{e}$ \\
\hline DM 66168 RSF IPRO & $0,12 \mathrm{~b}$ & $0,68 \mathrm{e}$ & 202000 & 16,73 a & $1603 \mathrm{~g}$ \\
\hline DM $68 \mathrm{i} 69$ IPRO & $0,13 \mathrm{~b}$ & $0,72 \mathrm{~d}$ & 151333 & 16,10 a & $2498 \mathrm{~d}$ \\
\hline HO Corumbá IPRO & 0,15 a & $0,80 \mathrm{c}$ & 273333 & 16,80 a & 2177 e \\
\hline HO Cristalino IPRO & $0,09 \mathrm{c}$ & $0,85 \mathrm{c}$ & 184667 & $14,87 \mathrm{~b}$ & 2077 e \\
\hline HO Maracaí IPRO & 0,14 a & $0,79 \mathrm{~d}$ & 147333 & $13,60 \mathrm{c}$ & $1768 \mathrm{f}$ \\
\hline HO Iguaçu IPRO & 0,16 a & $0,83 \mathrm{c}$ & 342667 & $11,77 \mathrm{~d}$ & $1528 \mathrm{~h}$ \\
\hline LG 60158 IPRO & $0,10 \mathrm{c}$ & $0,64 \mathrm{e}$ & 156000 & 16,13 a & 3172 a \\
\hline LG 60162 IPRO & $0,11 \mathrm{~b}$ & $0,60 \mathrm{f}$ & 256000 & $14,97 \mathrm{~b}$ & 2079 e \\
\hline LG 60174 IPRO & $0,12 \mathrm{~b}$ & $0,85 \mathrm{c}$ & 256667 & $13,33 \mathrm{c}$ & $1407 \mathrm{~h}$ \\
\hline LG 60179 IPRO & $0,12 \mathrm{~b}$ & $0,91 \mathrm{~b}$ & 382667 & $13,70 \mathrm{c}$ & $2903 \mathrm{~b}$ \\
\hline NS 6906 IPRO & $0,13 \mathrm{~b}$ & $0,75 \mathrm{~d}$ & 191333 & 16,63 a & $2669 \mathrm{c}$ \\
\hline NS 7667 IPRO & 0,16 a & $0,75 \mathrm{~d}$ & 227333 & $15,20 \mathrm{~b}$ & $2403 \mathrm{~d}$ \\
\hline NS 6700 IPRO & $0,13 \mathrm{~b}$ & $0,79 \mathrm{~d}$ & 192667 & $12,77 \mathrm{~d}$ & $2759 \mathrm{~b}$ \\
\hline TMG 7067 IPRO & $0,12 \mathrm{~b}$ & $0,84 \mathrm{c}$ & 263333 & $14,03 \mathrm{c}$ & $1527 \mathrm{~h}$ \\
\hline BRS Valiosa RR & $0,12 \mathrm{~b}$ & $1,05 \mathrm{a}$ & 277333 & $13,83 \mathrm{c}$ & $1886 \mathrm{f}$ \\
\hline $1 \mathrm{XT}-\mathrm{I}$ & $0,09 \mathrm{c}$ & $0,75 \mathrm{~d}$ & 229333 & 17,50 a & $2857 \mathrm{~b}$ \\
\hline $2 \mathrm{XN}$ & $0,12 \mathrm{~b}$ & $0,75 \mathrm{~d}$ & 175333 & $15,07 \mathrm{~b}$ & $2862 \mathrm{~b}$ \\
\hline 3 YM-I & $0,13 \mathrm{~b}$ & $0,74 \mathrm{~d}$ & 330667 & $14,03 \mathrm{c}$ & $1868 \mathrm{f}$ \\
\hline 4 YB-I & $0,09 \mathrm{c}$ & $0,78 \mathrm{~d}$ & 190667 & $15,17 \mathrm{~b}$ & $1675 \mathrm{~g}$ \\
\hline $5 \mathrm{KW}$ & $0,10 \mathrm{c}$ & 0,82 c & 270667 & $12,47 \mathrm{~d}$ & $1440 \mathrm{~h}$ \\
\hline 6 WB-I & $0,13 \mathrm{~b}$ & $0,73 \mathrm{~d}$ & 306667 & $15,33 \mathrm{~b}$ & $2625 \mathrm{c}$ \\
\hline $\mathrm{CV}^{(2)}(\%)$ & 3,95 & 9,16 & & 5,90 & 4,05 \\
\hline
\end{tabular}

Nucleus, Edição Especial, 2019 
Tabela 04. Características agronômicas avaliadas em diferentes cultivares de soja, Colina, SP, 2019.

(Conclusão)

\begin{tabular}{|c|c|c|c|}
\hline Cultivar & $\begin{array}{c}\text { Índice de } \\
\text { acamamento }\end{array}$ & Valor agronômico & $\begin{array}{l}\text { Número de dias } \\
\text { para a maturação }\end{array}$ \\
\hline AS 3610 IPRO & $1,3 \mathrm{~b}$ & $2,0 \mathrm{c}$ & 123 \\
\hline AS 3590 IPRO & $1,3 \mathrm{~b}$ & $2,5 \mathrm{~b}$ & 125 \\
\hline AS 3680 IPRO & $1,3 \mathrm{~b}$ & $2,3 \mathrm{~b}$ & 122 \\
\hline AS 3730 IPRO & $1,8 \mathrm{a}$ & 3,0 a & 125 \\
\hline Caraíba Speed & $1,2 \mathrm{~b}$ & $2,5 \mathrm{~b}$ & 120 \\
\hline Caraíba Nitro & $1,0 \mathrm{~b}$ & $2,3 \mathrm{~b}$ & 121 \\
\hline 64i61RSF ${ }^{(1)}$ & $1,2 \mathrm{~b}$ & $2,0 \mathrm{c}$ & 124 \\
\hline DM 66168 RSF IPRO & $1,5 \mathrm{a}$ & $2,0 \mathrm{c}$ & 125 \\
\hline DM 68169 IPRO & $1,5 \mathrm{a}$ & $2,5 \mathrm{~b}$ & 125 \\
\hline HO Corumbá IPRO & $1,8 \mathrm{a}$ & $2,5 \mathrm{~b}$ & 124 \\
\hline HO Cristalino IPRO & $1,3 \mathrm{~b}$ & 3,2 a & 126 \\
\hline HO Maracaí IPRO & $1,5 \mathrm{a}$ & $2,2 \mathrm{c}$ & 125 \\
\hline HO Iguaçu IPRO & $1,0 \mathrm{~b}$ & $2,0 \mathrm{c}$ & 124 \\
\hline LG 60158 IPRO & $1,3 \mathrm{~b}$ & 3,0 a & 122 \\
\hline LG 60162 IPRO & $1,2 \mathrm{~b}$ & $2,0 \mathrm{c}$ & 124 \\
\hline LG 60174 IPRO & $1,2 \mathrm{~b}$ & $2,0 \mathrm{c}$ & 126 \\
\hline LG 60179 IPRO & $1,0 \mathrm{~b}$ & 3,0 a & 126 \\
\hline NS 6906 IPRO & $1,2 \mathrm{~b}$ & 3,0 a & 122 \\
\hline NS 7667 IPRO & $1,7 \mathrm{a}$ & 2,7 a & 122 \\
\hline NS 6700 IPRO & $1,5 \mathrm{a}$ & 3,0 a & 126 \\
\hline TMG 7067 IPRO & $1,3 \mathrm{~b}$ & $2,0 \mathrm{c}$ & 123 \\
\hline BRS Valiosa RR & $1,7 \mathrm{a}$ & $2,2 \mathrm{c}$ & 127 \\
\hline $1 \mathrm{XT}-\mathrm{I}$ & $1,7 \mathrm{a}$ & 3,0 a & 120 \\
\hline $2 \mathrm{XN}$ & $1,0 \mathrm{~b}$ & 3,0 a & 122 \\
\hline 3 YM-I & $1,2 \mathrm{~b}$ & $2,3 \mathrm{~b}$ & 123 \\
\hline 4 YB-I & $1,2 \mathrm{~b}$ & $2,2 \mathrm{c}$ & 122 \\
\hline $5 \mathrm{KW}$ & $1,2 \mathrm{~b}$ & $1,8 \mathrm{c}$ & 124 \\
\hline 6 WB-I & $1,5 \mathrm{a}$ & 3,0 a & 120 \\
\hline $\mathrm{CV}^{(2)}(\%)$ & 23,06 & 11,28 & \\
\hline
\end{tabular}

A produtividade média de grãos foi de $2099 \mathrm{~kg} \mathrm{ha}^{-1}$. Enfatiza-se que, como em Andradina e Araçatuba, a evapotranspiração também foi bem acentuada nos meses de dezembro e, principalmente, em janeiro, fase de enchimento de grãos, no qual se registrou uma evapotranspiração de $155 \mathrm{~mm}$ e uma 
precipitação de apenas $55,9 \mathrm{~mm}$ e, durante os três meses, temperaturas máximas acima de $35^{\circ} \mathrm{C}$ e temperaturas médias acima de $24^{\circ} \mathrm{C}$ (Figuras 1,2 e 3 ).

As características agronômicas da cultura da soja, em Jaboticabal, estão demonstradas nas Tabelas 06 e 07. Assim como em Araçatuba e Colina, as diferentes cultivares também diferiram entre si $(\mathrm{p}<0,05)$ em relação à altura de inserção da primeira vagem, altura de plantas, massa de cem grãos e produtividade de grãos, no entanto, a produtividade média de grãos foi de $3453 \mathrm{~kg} \mathrm{ha}^{-1}$, superior à média do Estado de São Paulo que foi de $3039 \mathrm{~kg} \mathrm{ha}^{-1}$, segundo o oitavo levantamento da safra de grãos (CONAB, 2019).

Tabela 05. Características agronômicas avaliadas em diferentes cultivares de soja, Jaboticabal, SP, 2019.

(Continua)

\begin{tabular}{|c|c|c|c|c|c|}
\hline Cultivar & $\begin{array}{l}\text { Altura de } \\
\text { inserção }^{(6)}\end{array}$ & $\begin{array}{c}\text { Altura de } \\
\text { plantas }\end{array}$ & $\begin{array}{c}\text { Estande } \\
\text { final } \\
\text { (ha) }\end{array}$ & $\begin{array}{l}\text { Massa de cem } \\
\text { grãos } \\
\text { (g) }\end{array}$ & $\begin{array}{l}\text { Produtividade } \\
\text { de grãos } \\
\left(\mathrm{kg} \mathrm{ha}^{-1}\right)\end{array}$ \\
\hline AS 3610 IPRO & $0,14 b^{(7)}$ & $0,76 \mathrm{c}$ & 4,5 & $14,00 \mathrm{c}$ & $2825 \mathrm{~b}$ \\
\hline AS 3590 IPRO & $0,14 \mathrm{~b}$ & $0,81 \mathrm{~b}$ & 4,0 & $15,13 \mathrm{c}$ & 3753 a \\
\hline AS 3680 IPRO & $0,14 \mathrm{~b}$ & $0,95 \mathrm{a}$ & 4,8 & $13,99 \mathrm{c}$ & $2891 \mathrm{~b}$ \\
\hline AS 3730 IPRO & 0,17 a & $0,93 \mathrm{a}$ & 4,5 & $16,24 \mathrm{~b}$ & $3368 \mathrm{~b}$ \\
\hline Caraíba Speed & $0,13 \mathrm{~b}$ & $0,83 \mathrm{~b}$ & 5,0 & $11,87 \mathrm{~d}$ & $3347 \mathrm{~b}$ \\
\hline Caraíba Nitro & $0,12 \mathrm{~b}$ & $0,74 \mathrm{c}$ & 4,7 & $14,36 \mathrm{c}$ & $2896 \mathrm{~b}$ \\
\hline 64i61RSF ${ }^{(1)}$ & $0,15 \mathrm{~b}$ & 0,93 a & 4,7 & $14,13 \mathrm{c}$ & 4045 a \\
\hline DM 66i68 RSF IPRO & $0,14 \mathrm{~b}$ & $0,83 \mathrm{~b}$ & 4,5 & $16,49 \mathrm{~b}$ & $3293 \mathrm{~b}$ \\
\hline $68 \mathrm{i} 70 \mathrm{RSF}^{(2)}$ & 0,17 a & $0,86 \mathrm{~b}$ & 4,3 & $16,67 \mathrm{~b}$ & $3476 \mathrm{~b}$ \\
\hline $68 \mathrm{i} 68 \mathrm{RSF}^{(3)}$ & $0,14 \mathrm{~b}$ & $0,87 \mathrm{~b}$ & 4,5 & $17,06 \mathrm{~b}$ & 3651 a \\
\hline DM 68 i69 IPRO & $0,14 \mathrm{~b}$ & $0,77 \mathrm{c}$ & 4,8 & $16,42 \mathrm{~b}$ & 3979 a \\
\hline $74 \mathrm{i} 77 \mathrm{RSF}^{(4)}$ & $0,13 \mathrm{~b}$ & $1,02 \mathrm{a}$ & 4,7 & $14,42 \mathrm{c}$ & 3708 a \\
\hline HO Aporé IPRO & $0,13 \mathrm{~b}$ & $1,00 \mathrm{a}$ & 4,3 & $14,28 \mathrm{c}$ & 4219 a \\
\hline HO Corumbá IPRO & 0,17 a & $0,89 \mathrm{~b}$ & 4,5 & $15,88 \mathrm{~b}$ & 3944 a \\
\hline HO Cristalino IPRO & 0,17 a & $1,01 \mathrm{a}$ & 4,3 & $12,75 \mathrm{~d}$ & $3238 \mathrm{~b}$ \\
\hline HO Maracaí IPRO & $0,20 \mathrm{a}$ & $1,04 \mathrm{a}$ & 5,0 & $15,07 \mathrm{c}$ & $3296 \mathrm{~b}$ \\
\hline HO Iguaçu IPRO & $0,14 \mathrm{~b}$ & 0,91 a & 4,8 & $11,61 \mathrm{~d}$ & $3245 \mathrm{~b}$ \\
\hline LG 60158 IPRO & $0,11 \mathrm{~b}$ & $0,65 \mathrm{c}$ & 4,7 & $14,09 \mathrm{c}$ & $3225 \mathrm{~b}$ \\
\hline LG 60162 IPRO & $0,13 \mathrm{~b}$ & $0,78 \mathrm{c}$ & 4,8 & $14,57 \mathrm{c}$ & 3655 a \\
\hline LG 60174 IPRO & $0,15 \mathrm{~b}$ & $0,86 \mathrm{~b}$ & 4,8 & $13,71 \mathrm{c}$ & 3562 a \\
\hline LG 60179 IPRO & $0,14 \mathrm{~b}$ & 0,99 a & 5,0 & $11,35 \mathrm{~d}$ & $3210 \mathrm{~b}$ \\
\hline NS 6906 IPRO & 0,18 a & $0,86 \mathrm{~b}$ & 4,8 & $14,97 \mathrm{c}$ & $2938 \mathrm{~b}$ \\
\hline NS 7709 IPRO & 0,17 a & 0,98 a & 4,7 & $15,67 \mathrm{~b}$ & $3296 \mathrm{~b}$ \\
\hline NS 7667 IPRO & $0,15 \mathrm{~b}$ & $0,94 \mathrm{a}$ & 4,7 & $13,94 \mathrm{c}$ & $3459 \mathrm{~b}$ \\
\hline NS 6700 IPRO & 0,17 a & $1,01 \mathrm{a}$ & 4,7 & $15,14 \mathrm{c}$ & 3794 a \\
\hline
\end{tabular}


Tabela 05. Características agronômicas avaliadas em diferentes cultivares de soja, Jaboticabal, SP, 2019.

(Conclusão)

\begin{tabular}{|c|c|c|c|c|c|}
\hline Cultivar & $\begin{array}{l}\text { Altura de } \\
\text { inserção }^{(6)}\end{array}$ & $\begin{array}{l}\text { Altura de } \\
\text { plantas }\end{array}$ & $\begin{array}{c}\text { Estande } \\
\text { final } \\
\text { (ha) }\end{array}$ & $\begin{array}{c}\text { Massa de cem } \\
\text { grãos } \\
\text { (g) }\end{array}$ & $\begin{array}{c}\text { Produtividade } \\
\text { de grãos } \\
\left(\mathrm{kg} \mathrm{ha}^{-1}\right)\end{array}$ \\
\hline TEC 6702 IPRO & $0,14 \mathrm{~b}$ & $0,81 \mathrm{~b}$ & 4,7 & 18,36 a & 3688 a \\
\hline TMG 7067 IPRO & $0,15 \mathrm{~b}$ & $0,90 \mathrm{~b}$ & 4,7 & $16,28 \mathrm{~b}$ & 3635 a \\
\hline BRS Valiosa RR & $0,17 \mathrm{a}$ & $1,05 \mathrm{a}$ & 4,8 & $14,28 \mathrm{c}$ & $2542 b$ \\
\hline $1 \mathrm{XT}-\mathrm{I}$ & 0,16 a & 0,96 a & 4,3 & 17,91 a & $3229 \mathrm{~b}$ \\
\hline $2 \mathrm{XN}$ & $0,14 \mathrm{~b}$ & $0,84 \mathrm{~b}$ & 4,5 & $16,92 \mathrm{~b}$ & 3865 a \\
\hline $3 \mathrm{YM}-\mathrm{I}$ & $0,15 \mathrm{~b}$ & $0,90 \mathrm{~b}$ & 4,7 & $15,59 \mathrm{~b}$ & 4154 a \\
\hline 4 YB-I & $0,13 \mathrm{~b}$ & $0,77 \mathrm{c}$ & 5,0 & $13,82 \mathrm{c}$ & 3809 a \\
\hline $5 \mathrm{KW}$ & 0,17 a & $1,11 \mathrm{a}$ & 4,8 & $14,96 \mathrm{c}$ & $2978 \mathrm{~b}$ \\
\hline $6 \mathrm{WB}-\mathrm{I}$ & 0,16 a & $0,84 \mathrm{~b}$ & 4,7 & $14,67 \mathrm{c}$ & $3193 \mathrm{~b}$ \\
\hline $\mathrm{CV}^{(5)}(\%)$ & 15,06 & 8,43 & 8,46 & 6,54 & 12,48 \\
\hline
\end{tabular}

Tabela 06. Características agronômicas avaliadas em diferentes cultivares de soja Jaboticabal, SP, 2019.

(Continua)

\begin{tabular}{|c|c|c|c|}
\hline Cultivar & $\begin{array}{c}\text { Índice de } \\
\text { acamamento }\end{array}$ & Valor agronômico & $\begin{array}{c}\text { Número de dias } \\
\text { para a maturação }\end{array}$ \\
\hline AS 3610 IPRO & $1,5 c^{(6)}$ & $3,2 \mathrm{~b}$ & 116 \\
\hline AS 3590 IPRO & $1,0 \mathrm{~d}$ & $4,3 \mathrm{a}$ & 121 \\
\hline AS 3680 IPRO & $1,8 \mathrm{~b}$ & $3,2 \mathrm{~b}$ & 116 \\
\hline AS 3730 IPRO & $1,0 \mathrm{~d}$ & $4,2 \mathrm{a}$ & 121 \\
\hline Caraíba Speed & $1,0 \mathrm{~d}$ & $4,0 \mathrm{a}$ & 117 \\
\hline Caraíba Nitro & $1,0 \mathrm{~d}$ & $3,3 \mathrm{~b}$ & 116 \\
\hline $64 \mathrm{i} 61 \mathrm{RSF}^{(1)}$ & $1,3 \mathrm{c}$ & $4,3 \mathrm{a}$ & 120 \\
\hline DM 66i68 RSF IPRO & $1,0 \mathrm{~d}$ & 3,8 a & 118 \\
\hline $68 \mathrm{i} 70 \mathrm{RSF}^{(2)}$ & $1,2 \mathrm{~d}$ & $3,5 \mathrm{~b}$ & 118 \\
\hline $68 \mathrm{i} 68 \mathrm{RSF}^{(3)}$ & $1,2 \mathrm{~d}$ & $3,2 \mathrm{~b}$ & 118 \\
\hline DM 68 i69 IPRO & $1,3 \mathrm{c}$ & $3,0 \mathrm{~b}$ & 117 \\
\hline $74 \mathrm{i} 77 \mathrm{RSF}^{(4)}$ & $1,3 \mathrm{c}$ & $4,2 \mathrm{a}$ & 120 \\
\hline HO Aporé IPRO & $1,3 \mathrm{c}$ & 4,2 a & 120 \\
\hline HO Corumbá IPRO & $1,8 \mathrm{~b}$ & $3,2 \mathrm{~b}$ & 120 \\
\hline HO Cristalino IPRO & $1,3 \mathrm{c}$ & $4,7 \mathrm{a}$ & 131 \\
\hline
\end{tabular}


Tabela 06. Características agronômicas avaliadas em diferentes cultivares de soja Jaboticabal, SP, 2019.

(Conclusão)

\begin{tabular}{|c|c|c|c|}
\hline Cultivar & $\begin{array}{c}\text { Índice de } \\
\text { acamamento }\end{array}$ & Valor agronômico & $\begin{array}{c}\text { Número de dias } \\
\text { para a maturação }\end{array}$ \\
\hline HO Maracaí IPRO & $2,2 \mathrm{a}$ & $3,5 \mathrm{~b}$ & 126 \\
\hline HO Iguaçu IPRO & $1,2 \mathrm{~d}$ & $3,7 \mathrm{~b}$ & 120 \\
\hline LG 60158 IPRO & $1,0 \mathrm{~d}$ & $3,0 \mathrm{~b}$ & 116 \\
\hline LG 60162 IPRO & $1,0 \mathrm{~d}$ & 4,3 a & 119 \\
\hline LG 60174 IPRO & $1,0 \mathrm{~d}$ & $3,5 \mathrm{~b}$ & 124 \\
\hline LG 60179 IPRO & $1,0 \mathrm{~d}$ & 4,3 a & 127 \\
\hline NS 6906 IPRO & $1,7 \mathrm{~b}$ & $2,8 \mathrm{~b}$ & 118 \\
\hline NS 7709 IPRO & $1,5 \mathrm{c}$ & $4,0 \quad \mathrm{a}$ & 121 \\
\hline NS 7667 IPRO & $1,5 \mathrm{c}$ & 4,0 a & 125 \\
\hline NS 6700 IPRO & $1,5 \mathrm{c}$ & $4,2 \mathrm{a}$ & 125 \\
\hline TEC 6702 IPRO & $1,0 \mathrm{~d}$ & $3,0 \mathrm{~b}$ & 115 \\
\hline TMG 7067 IPRO & $1,3 \mathrm{c}$ & $4,3 \mathrm{a}$ & 123 \\
\hline BRS Valiosa RR & $1,3 \mathrm{c}$ & $4,7 \mathrm{a}$ & 130 \\
\hline $1 \mathrm{XT}-\mathrm{I}$ & $1,2 \mathrm{~d}$ & $4,0 \quad \mathrm{a}$ & 118 \\
\hline $2 \mathrm{XN}$ & $1,2 \mathrm{~d}$ & 3,8 a & 123 \\
\hline 3 YM-I & $1,0 \mathrm{~d}$ & $4,2 \mathrm{a}$ & 120 \\
\hline 4 YB-I & $1,0 \mathrm{~d}$ & $4,0 \quad \mathrm{a}$ & 117 \\
\hline $5 \mathrm{KW}$ & $2,3 \mathrm{a}$ & $4,0 \mathrm{a}$ & 121 \\
\hline $6 \mathrm{WB}-\mathrm{I}$ & $1,8 \mathrm{~b}$ & 3,8 a & 119 \\
\hline $\mathrm{CV}^{(5)}(\%)$ & 18,03 & 12,2 & \\
\hline
\end{tabular}

Na Tabela 08 estão demonstradas as características agronômicas da cultura da soja de Manduri. As diferentes cultivares diferiram entre si $(\mathrm{p}<0,05)$ em relação à altura de plantas e produtividade de grãos, no entanto, não houve diferença entre as cultivares em relação à altura de inserção da primeira vagem, provavelmente devido ao alto coeficiente de variação apresentado. A média da produtividade de grãos foi de $3093 \mathrm{~kg} \mathrm{ha}^{-1}$, também superior à média do Estado de São Paulo de $3039 \mathrm{~kg} \mathrm{ha}^{-1}$ (CONAB, 2019). 
Tabela 07. Características agronômicas avaliadas em diferentes cultivares de soja, Manduri, SP, 2019.

\begin{tabular}{|c|c|c|c|c|}
\hline Cultivar & $\begin{array}{l}\text { Altura de } \\
\text { inserção }^{(6)}\end{array}$ & $\begin{array}{c}\text { Altura de } \\
\text { plantas }\end{array}$ & $\begin{array}{c}\text { Estande final } \\
\text { (ha) }\end{array}$ & $\begin{array}{c}\text { Produtividade } \\
\text { de grãos } \\
\left(\mathrm{kg} \mathrm{ha}^{-1}\right)\end{array}$ \\
\hline AS 3610 IPRO & $0,10^{(\mathrm{ns})}$ & $0,65 b^{(7)}$ & 260000 & $2757 \mathrm{~b}$ \\
\hline AS 3590 IPRO & 0,11 & $0,62 \mathrm{~b}$ & 233000 & 3918 a \\
\hline AS 3680 IPRO & 0,12 & 0,74 a & 215500 & $2850 \mathrm{~b}$ \\
\hline Caraíba Speed & 0,10 & $0,67 \mathrm{~b}$ & 273500 & $2791 \mathrm{~b}$ \\
\hline Caraíba Nitro & 0,26 & $0,59 \mathrm{~b}$ & 255000 & $2807 \mathrm{~b}$ \\
\hline 64i61RSF ${ }^{(1)}$ & 0,11 & $0,65 \mathrm{~b}$ & 207000 & 3362 a \\
\hline DM 66i68 RSF IPRO & 0,11 & $0,65 \mathrm{~b}$ & 253500 & 3466 a \\
\hline $68 \mathrm{i} 70 \mathrm{RSF}^{(2)}$ & 0,07 & $0,64 \mathrm{~b}$ & 117000 & $2869 \mathrm{~b}$ \\
\hline $68 \mathrm{i} 68 \mathrm{RSF}^{(3)}$ & 0,06 & $0,58 \mathrm{~b}$ & 184000 & $2812 \mathrm{~b}$ \\
\hline DM $68 \mathrm{i} 69$ IPRO & 0,08 & $0,64 \mathrm{~b}$ & 240000 & 3418 a \\
\hline $74 \mathrm{i} 77 \mathrm{RSF}^{(4)}$ & 0,07 & 0,75 a & 254500 & 3870 a \\
\hline HO Aporé IPRO & 0,09 & 0,72 a & 144667 & $2944 \mathrm{~b}$ \\
\hline HO Corumbá IPRO & 0,11 & $0,66 \mathrm{~b}$ & 190000 & 3421 a \\
\hline HO Cristalino IPRO & 0,09 & 0,83 a & 187000 & 3248 a \\
\hline HO Maracaí IPRO & 0,13 & 0,78 a & 201000 & 3290 a \\
\hline HO Iguaçu IPRO & 0,08 & $0,58 \mathrm{~b}$ & 149000 & $2419 \mathrm{~b}$ \\
\hline LG 60158 IPRO & 0,12 & $0,63 \mathrm{~b}$ & 216000 & $2505 \mathrm{~b}$ \\
\hline LG 60162 IPRO & 0,11 & $0,62 \mathrm{~b}$ & 239000 & 3768 a \\
\hline LG 60174 IPRO & 0,10 & 0,71 a & 232500 & 3499 a \\
\hline LG 60179 IPRO & 0,14 & 0,85 a & 369950 & $2987 \mathrm{~b}$ \\
\hline NS 7709 IPRO & 0,09 & 0,74 a & 255500 & 3319 a \\
\hline NS 7667 IPRO & 0,10 & 0,76 a & 270000 & $3004 \mathrm{~b}$ \\
\hline NS 6700 IPRO & 0,10 & $0,74 \mathrm{a}$ & 258500 & $3480 \mathrm{a}$ \\
\hline TEC 6702 IPRO & 0,09 & $0,62 \mathrm{~b}$ & 209500 & $3028 \mathrm{~b}$ \\
\hline TMG 7067 IPRO & 0,12 & $0,67 \mathrm{~b}$ & 218500 & 3423 a \\
\hline BRS 284 & 0,09 & 0,79 a & 229500 & 3434 a \\
\hline BRS 511 & 0,09 & $0,72 \mathrm{a}$ & 152500 & 3374 a \\
\hline $1 \mathrm{XT}-\mathrm{I}$ & 0,09 & $0,60 \mathrm{~b}$ & 160667 & $1909 \mathrm{c}$ \\
\hline 3 YM-I & 0,10 & $0,61 \mathrm{~b}$ & 199000 & 3430 a \\
\hline 4 YB-I & 0,08 & $0,61 \mathrm{~b}$ & 150500 & $2511 \mathrm{~b}$ \\
\hline $5 \mathrm{KW}$ & 0,10 & $0,72 \mathrm{a}$ & 100000 & $1919 \mathrm{c}$ \\
\hline $6 \mathrm{WB}-\mathrm{I}$ & 0,09 & $0,69 \mathrm{~b}$ & 115000 & 3155 a \\
\hline $\mathrm{CV}^{(5)}(\%)$ & 66,97 & 9,80 & & 13,30 \\
\hline
\end{tabular}


As características agronômicas da cultura da soja, em Pindorama, estão demonstradas nas Tabelas 09 e 10. As diferentes cultivares diferiram entre si $(\mathrm{p}<0,05)$ em relação à altura de plantas, massa de cem grãos e produtividade de grãos, no entanto, como em Manduri, não houve diferença entre as cultivares em relação à altura de inserção da primeira vagem. A média de produtividade de grãos em Pindorama foi a maior entre os oito locais avaliados $\left(4043 \mathrm{~kg} \mathrm{ha}^{-1}\right)$.

Tabela 08. Características agronômicas avaliadas em diferentes cultivares de soja, Pindorama, SP, 2019.

(Continua)

\begin{tabular}{|c|c|c|c|c|c|}
\hline Cultivar & $\begin{array}{l}\text { Altura de } \\
\text { inserção }^{(6)}\end{array}$ & $\begin{array}{l}\text { Altura de } \\
\text { plantas }\end{array}$ & $\begin{array}{c}\text { Estande } \\
\text { final } \\
\text { (ha) }\end{array}$ & $\begin{array}{c}\text { Massa de cem } \\
\text { grãos } \\
(\mathrm{g})\end{array}$ & $\begin{array}{c}\text { Produtividade de } \\
\text { grãos } \\
\left(\mathrm{kg} \mathrm{ha}^{-1}\right)\end{array}$ \\
\hline AS 3610 IPRO & $0,15^{(\mathrm{ns})}$ & $0,71 b^{(7)}$ & 364000 & $15,55 \mathrm{e}$ & $2887 \mathrm{f}$ \\
\hline AS 3590 IPRO & 0,12 & $0,64 \mathrm{~b}$ & 223889 & 21,53 a & 3508 e \\
\hline AS 3680 IPRO & 0,13 & 0,80 a & 363556 & $15,67 \mathrm{e}$ & 3127 e \\
\hline Caraíba Speed & 0,17 & $0,70 \mathrm{~b}$ & 255556 & $11,77 \mathrm{~h}$ & $2767 \mathrm{f}$ \\
\hline Caraíba Nitro & 0,12 & $0,70 \mathrm{~b}$ & 378889 & $13,43 \mathrm{~g}$ & $2654 \mathrm{f}$ \\
\hline 64i61RSF ${ }^{(1)}$ & 0,13 & 0,81 a & 277778 & $16,00 \mathrm{~d}$ & $5080 \mathrm{~b}$ \\
\hline DM 66i68 RSF IPRO & 0,12 & $0,65 \mathrm{~b}$ & 226667 & $17,74 \mathrm{c}$ & 3529 e \\
\hline $68 \mathrm{i} 70 \mathrm{RSF}^{(2)}$ & 0,12 & $0,68 \mathrm{~b}$ & 275667 & $15,06 \mathrm{e}$ & 3486 e \\
\hline $68 \mathrm{i} 68 \mathrm{RSF}^{(3)}$ & 0,14 & 0,78 a & 367111 & $14,94 \mathrm{e}$ & $4186 \mathrm{c}$ \\
\hline DM 68169 IPRO & 0,14 & $0,68 \mathrm{~b}$ & 308889 & $16,50 \mathrm{~d}$ & $3719 \mathrm{~d}$ \\
\hline 74i77 $\mathrm{RSF}^{(4)}$ & 0,14 & 0,78 a & 334667 & $15,21 \mathrm{e}$ & $4401 \mathrm{c}$ \\
\hline HO Aporé IPRO & 0,14 & 0,84 a & 249000 & 20,69 a & 5650 a \\
\hline HO Corumbá IPRO & 0,14 & $0,76 \mathrm{~b}$ & 339889 & $17,75 \mathrm{c}$ & 6050 a \\
\hline HO Cristalino IPRO & 0,21 & 0,84 a & 296667 & $14,56 \mathrm{f}$ & $3824 \mathrm{~d}$ \\
\hline HO Maracaí IPRO & 0,21 & 0,85 a & 320000 & $16,47 \mathrm{~d}$ & $3652 \mathrm{~d}$ \\
\hline HO Iguaçu IPRO & 0,15 & 0,82 a & 408667 & $13,57 \mathrm{~g}$ & $3892 \mathrm{~d}$ \\
\hline LG 60158 IPRO & 0,12 & $0,71 \mathrm{~b}$ & 296667 & $15,89 \mathrm{~d}$ & 3468 e \\
\hline LG 60162 IPRO & 0,16 & $0,71 \mathrm{~b}$ & 345667 & $15,51 \mathrm{e}$ & $3946 \mathrm{~d}$ \\
\hline LG 60174 IPRO & 0,13 & $0,74 \mathrm{~b}$ & 361000 & $17,06 \mathrm{~d}$ & $4404 \mathrm{c}$ \\
\hline LG 60179 IPRO & 0,15 & 0,87 a & 370333 & $16,51 \mathrm{~d}$ & $4418 \mathrm{c}$ \\
\hline NS 6906 IPRO & 0,16 & 0,85 a & 286667 & $16,30 \mathrm{~d}$ & $4144 \mathrm{c}$ \\
\hline NS 7709 IPRO & 0,18 & 0,88 a & 278667 & $18,75 \mathrm{~b}$ & $3771 \mathrm{~d}$ \\
\hline NS 7667 IPRO & 0,16 & $0,74 \mathrm{~b}$ & 236333 & $16,25 \mathrm{~d}$ & 6148 a \\
\hline NS 6700 IPRO & 0,17 & 0,85 a & 270222 & $16,53 \mathrm{~d}$ & 6247 a \\
\hline TEC 6702 IPRO & 0,13 & 0,78 a & 326111 & $14,54 \mathrm{f}$ & $3802 \mathrm{~d}$ \\
\hline TMG 7067 IPRO & 0,15 & $0,76 \mathrm{~b}$ & 344667 & $15,31 \mathrm{e}$ & $3717 \mathrm{~d}$ \\
\hline 1 XT-I & 0,12 & $0,75 \mathrm{~b}$ & 259444 & $15,15 \mathrm{e}$ & $4430 \mathrm{c}$ \\
\hline $2 \mathrm{XN}$ & 0,13 & $0,74 \mathrm{~b}$ & 271667 & $16,17 \mathrm{~d}$ & $3646 \mathrm{~d}$ \\
\hline
\end{tabular}


Tabela 08. Características agronômicas avaliadas em diferentes cultivares de soja, Pindorama, SP, 2019.

(Conclusão)

\begin{tabular}{|c|c|c|c|c|c|}
\hline Cultivar & $\begin{array}{l}\text { Altura de } \\
\text { inserção }^{(6)}\end{array}$ & $\begin{array}{l}\text { Altura de } \\
\text { plantas }\end{array}$ & $\begin{array}{c}\text { Estande } \\
\text { final } \\
\text { (ha) }\end{array}$ & $\begin{array}{l}\text { Massa de cem } \\
\text { grãos } \\
\text { (g) }\end{array}$ & $\begin{array}{c}\text { Produtividade de } \\
\text { grãos } \\
\left(\mathrm{kg} \mathrm{ha}^{-1}\right)\end{array}$ \\
\hline 3 YM-I & 0,15 & $0,69 \mathrm{~b}$ & 277778 & $16,77 \mathrm{~d}$ & $4787 \mathrm{~b}$ \\
\hline 4 YB-I & 0,12 & $0,73 \mathrm{~b}$ & 347222 & $16,61 \mathrm{~d}$ & 3375 e \\
\hline $5 \mathrm{KW}$ & 0,14 & 0,84 a & 301333 & $14,91 \mathrm{e}$ & $3226 \mathrm{e}$ \\
\hline $6 \mathrm{WB}-\mathrm{I}$ & 0,18 & 0,83 a & 320889 & $15,05 \mathrm{e}$ & 3430 e \\
\hline $\mathrm{CV}^{(5)}(\%)$ & 20,88 & 8,99 & & 3,49 & 10,17 \\
\hline
\end{tabular}

Tabela 09. Características agronômicas avaliadas em diferentes cultivares de soja, Pindorama, SP, 2019.

(Continua)

\section{Cultivar}

AS 3610 IPRO

AS 3590 IPRO

AS 3680 IPRO

Caraíba Speed

Caraíba Nitro

64i61RSF ${ }^{(1)}$

DM 66 i68 RSF IPRO

$68 \mathrm{i} 70 \mathrm{RSF}^{(2)}$

$68 \mathrm{i} 68 \mathrm{RSF}^{(3)}$

DM 68 i69 IPRO

$74 \mathrm{i} 77 \mathrm{RSF}^{(4)}$

HO Aporé IPRO

HO Corumbá IPRO

HO Cristalino IPRO

HO Maracaí IPRO

HO Iguaçu IPRO

LG 60158 IPRO

LG 60162 IPRO

LG 60174 IPRO

LG 60179 IPRO

\section{Número de dias até}

o pleno florescimento

49

48

48

47

47

47

47

47

47

47

46

46

47

52

49

47

47

49

49

49

\section{Número de dias \\ para a maturação}

105

105

102

102

102

105

105

105

102

103

109

109

105

121

102

102

99

113

115

121 
Tabela 09. Características agronômicas avaliadas em diferentes cultivares de soja, Pindorama, SP, 2019.

Conclusão)

\begin{tabular}{lcc}
\hline Cultivar & $\begin{array}{c}\text { Número de dias até } \\
\text { o pleno florescimento }\end{array}$ & $\begin{array}{c}\text { Número de dias } \\
\text { para a maturação }\end{array}$ \\
NS 6906 IPRO & 47 & 105 \\
NS 7709 IPRO & 50 & 105 \\
NS 7667 IPRO & 49 & 115 \\
NS 6700 IPRO & 48 & 105 \\
TEC 6702 IPRO & 47 & 103 \\
TMG 7067 IPRO & 47 & 102 \\
1 XT-I & 47 & 102 \\
2 XN & 46 & 102 \\
3 YM-I & 48 & 102 \\
4 YB-I & 47 & 102 \\
5 KW & 47 & 107 \\
6 WB-I & 47 & 105 \\
\hline (1) Brasmax Fibra IPRO; ${ }^{(2)} \mathrm{Bran}^{-1}$ &
\end{tabular}

${ }^{(1)}$ Brasmax Fibra IPRO; ${ }^{(2)}$ Brasmax Ícone IPRO; ${ }^{(3)}$ Brasmax Única IPRO; ${ }^{(4)}$ Brasmax Foco IPRO.

Na Tabela 11 estão demonstradas as características agronômicas da cultura da soja de Riolândia. As diferentes cultivares também diferiram entre si $(p<0,05)$ em relação à altura de inserção da primeira vagem, altura de plantas, massa de cem grãos e produtividade de grãos. A média de produtividade de grãos foi de $2734 \mathrm{~kg} \mathrm{ha}^{-1}$.

Tabela 10. Características agronômicas avaliadas em diferentes cultivares de soja, Riolândia, SP, 2019.

(Continua)

\begin{tabular}{|c|c|c|c|c|c|c|}
\hline Cultivar & $\begin{array}{r}\text { Altura de } \\
\text { inserção }^{(6)}\end{array}$ & $\begin{array}{l}\text { Altura de } \\
\text { plantas }\end{array}$ & $\begin{array}{c}\text { Estande } \\
\text { final } \\
\text { (ha) }\end{array}$ & $\begin{array}{l}\text { Ciclo } \\
\text { (dias) }\end{array}$ & $\begin{array}{c}\text { Massa de } \\
\text { cem grãos } \\
\text { (g) }\end{array}$ & $\begin{array}{c}\text { Produtividade } \\
\text { de grãos } \\
\left(\mathrm{kg} \mathrm{ha}^{-1}\right)\end{array}$ \\
\hline AS 3610 IPRO & $0,10 c^{(7)}$ & $0,59 \mathrm{~b}$ & 216000 & 98 & $16,19 \mathrm{c}$ & $2376 \mathrm{~d}$ \\
\hline AS 3590 IPRO & $0,11 \mathrm{c}$ & $0,60 \mathrm{~b}$ & 239500 & 98 & $13,78 \mathrm{~g}$ & $2713 \mathrm{c}$ \\
\hline AS 3680 IPRO & $0,11 \mathrm{c}$ & $0,62 \mathrm{~b}$ & 258500 & 98 & $15,69 \mathrm{~d}$ & $2710 \mathrm{c}$ \\
\hline Caraíba Speed & $0,08 \mathrm{~d}$ & $0,57 \mathrm{~b}$ & 264000 & 98 & $14,45 \mathrm{f}$ & $2285 \mathrm{~d}$ \\
\hline Caraíba Nitro & $0,10 \mathrm{c}$ & $0,51 \mathrm{c}$ & 289000 & 98 & $14,65 \mathrm{e}$ & $2027 \mathrm{~d}$ \\
\hline $64 \mathrm{i} 61 \mathrm{RSF}^{(1)}$ & $0,10 \mathrm{c}$ & $0,61 \mathrm{~b}$ & 261000 & 107 & $13,43 \mathrm{~g}$ & $3173 \mathrm{~b}$ \\
\hline DM 66i68 RSF IPRO & $0,10 \mathrm{c}$ & $0,53 \mathrm{c}$ & 256000 & 107 & $16,30 \mathrm{c}$ & $2871 \mathrm{c}$ \\
\hline $68 \mathrm{i} 70 \mathrm{RSF}^{(2)}$ & $0,09 \mathrm{c}$ & $0,50 \mathrm{c}$ & 257333 & 107 & $17,48 \quad b$ & $2456 \mathrm{~d}$ \\
\hline $68 \mathrm{i} 68 \mathrm{RSF}^{(3)}$ & $0,10 \mathrm{c}$ & $0,61 \mathrm{~b}$ & 283500 & 111 & $16,80 \mathrm{c}$ & $2856 \mathrm{c}$ \\
\hline DM $68 i 69$ IPRO & $0,11 \mathrm{c}$ & $0,53 \mathrm{c}$ & 294000 & 107 & $14,39 \mathrm{f}$ & $2641 \mathrm{~d}$ \\
\hline
\end{tabular}


Tabela 10. Características agronômicas avaliadas em diferentes cultivares de soja, Riolândia, SP, 2019.

(Conclusão)

\begin{tabular}{|c|c|c|c|c|c|c|}
\hline Cultivar & $\begin{array}{r}\text { Altura de } \\
\text { inserção }^{(6)}\end{array}$ & $\begin{array}{l}\text { Altura de } \\
\text { plantas }\end{array}$ & $\begin{array}{c}\text { Estande } \\
\text { final } \\
\text { (ha) }\end{array}$ & $\begin{array}{l}\text { Ciclo } \\
\text { (dias) }\end{array}$ & $\begin{array}{c}\text { Massa de } \\
\text { cem grãos } \\
\text { (g) }\end{array}$ & $\begin{array}{c}\text { Produtividade } \\
\text { de grãos } \\
\left(\mathrm{kg} \mathrm{ha}^{-1}\right)\end{array}$ \\
\hline $74 \mathrm{i} 77 \mathrm{RSF}^{(4)}$ & $0,09 \mathrm{c}$ & $0,61 \mathrm{~b}$ & 261333 & 111 & $14,75 \mathrm{e}$ & $3057 \mathrm{c}$ \\
\hline HO Aporé IPRO & $0,09 \mathrm{c}$ & $0,61 \mathrm{~b}$ & 261500 & 117 & $13,55 \mathrm{~g}$ & 2916 c \\
\hline HO Corumbá IPRO & $0,11 \mathrm{~b}$ & $0,54 \mathrm{c}$ & 288667 & 111 & $16,46 \mathrm{c}$ & $3032 \mathrm{c}$ \\
\hline HO Cristalino IPRO & 0,13 a & 0,67 a & 242500 & 117 & $14,04 \mathrm{f}$ & $3076 \mathrm{c}$ \\
\hline HO Maracaí IPRO & 0,15 a & 0,71 a & 255500 & 117 & $17,03 \mathrm{~b}$ & 3634 a \\
\hline HO Iguaçu IPRO & $0,10 \mathrm{c}$ & $0,59 \mathrm{~b}$ & 298000 & 107 & $12,78 \mathrm{~h}$ & $2871 \mathrm{c}$ \\
\hline LG 60158 IPRO & $0,06 \mathrm{e}$ & $0,45 \mathrm{~d}$ & 253000 & 98 & $12,14 \mathrm{~h}$ & $2359 \mathrm{~d}$ \\
\hline LG 60162 IPRO & $0,10 \mathrm{c}$ & $0,50 \mathrm{c}$ & 266667 & 107 & $14,26 \mathrm{f}$ & $2822 \mathrm{c}$ \\
\hline LG 60174 IPRO & $0,10 \mathrm{c}$ & $0,56 \mathrm{~b}$ & 286000 & 114 & $14,91 \mathrm{e}$ & $3241 \mathrm{~b}$ \\
\hline LG 60179 IPRO & $0,11 \mathrm{c}$ & $0,70 \mathrm{a}$ & 277500 & 117 & $13,89 \mathrm{~g}$ & $2726 \mathrm{c}$ \\
\hline NS 6906 IPRO & $0,10 \mathrm{c}$ & $0,51 \mathrm{c}$ & 274000 & 98 & $15,02 \mathrm{e}$ & $2299 \mathrm{~d}$ \\
\hline NS 7709 IPRO & $0,12 \mathrm{~b}$ & $0,63 \mathrm{~b}$ & 246000 & 111 & $16,11 \mathrm{~d}$ & $2801 \mathrm{c}$ \\
\hline NS 7667 IPRO & $0,10 \mathrm{c}$ & $0,58 \mathrm{~b}$ & 249000 & 111 & $15,92 \mathrm{~d}$ & 3484 a \\
\hline NS 6700 IPRO & $0,12 \mathrm{~b}$ & $0,63 \mathrm{~b}$ & 266500 & 111 & $16,43 \mathrm{c}$ & 2946 c \\
\hline TEC 6702 IPRO & $0,11 \mathrm{~b}$ & $0,57 \mathrm{~b}$ & 253333 & 98 & 18,42 a & $2316 \mathrm{~d}$ \\
\hline TMG 7067 IPRO & $0,10 \mathrm{c}$ & $0,58 \mathrm{~b}$ & 254000 & 98 & $14,07 \mathrm{f}$ & $2368 \mathrm{~d}$ \\
\hline $1 \mathrm{XT}-\mathrm{I}$ & $0,13 \mathrm{~b}$ & $0,60 \mathrm{~b}$ & 253000 & 98 & $16,63 \mathrm{c}$ & $2633 \mathrm{~d}$ \\
\hline $2 \mathrm{XN}$ & $0,10 \mathrm{c}$ & $0,59 \mathrm{~b}$ & 270000 & 107 & $15,63 \mathrm{~d}$ & $2471 \mathrm{~d}$ \\
\hline 3 YM-I & $0,14 \mathrm{a}$ & $0,56 \mathrm{~b}$ & 212000 & 107 & $14,96 \mathrm{e}$ & $2857 \mathrm{c}$ \\
\hline 4 YB-I & $0,09 \mathrm{c}$ & $0,46 \mathrm{~d}$ & 279500 & 98 & $13,75 \mathrm{~g}$ & $2365 \mathrm{~d}$ \\
\hline $5 \mathrm{KW}$ & $0,12 \mathrm{~b}$ & $0,60 \mathrm{~b}$ & 242500 & 107 & $14,52 \mathrm{f}$ & $2350 \mathrm{~d}$ \\
\hline 6 WB-I & $0,11 \mathrm{c}$ & $0,64 \mathrm{~b}$ & 245500 & 98 & $14,83 \mathrm{e}$ & $2754 \mathrm{c}$ \\
\hline $\mathrm{CV}^{(5)}(\%)$ & 11,49 & 6,51 & & & 3,19 & 8,99 \\
\hline
\end{tabular}

As características agronômicas da cultura da soja, em Votuporanga, estão demonstradas na Tabela 12. As diferentes cultivares também diferiram entre si $(\mathrm{p}<0,05)$ em relação à altura de inserção da primeira vagem, altura de plantas, massa de cem grãos e produtividade de grãos. A média de produtividade de grãos foi de $3881 \mathrm{~kg} \mathrm{ha}^{-1}$, também superior à média do Estado de São Paulo de $3039 \mathrm{~kg} \mathrm{ha}^{-1}$ (CONAB, 2019). 
Tabela 11. Características agronômicas avaliadas em diferentes cultivares de soja, Votuporanga, SP, 2019.

\begin{tabular}{|c|c|c|c|c|c|c|}
\hline Cultivar & $\begin{array}{l}\text { Altura de } \\
\text { inserçãa }^{(6)}\end{array}$ & $\begin{array}{l}\text { Altura de } \\
\text { plantas }\end{array}$ & $\begin{array}{c}\text { Estande } \\
\text { final } \\
\text { (ha) }\end{array}$ & $\begin{array}{l}\text { Ciclo } \\
\text { (dias) }\end{array}$ & $\begin{array}{c}\text { Massa de } \\
\text { cem grãos } \\
\text { (g) }\end{array}$ & $\begin{array}{c}\text { Produtividade } \\
\text { de grãos } \\
\left(\text { kg ha }^{-1}\right)\end{array}$ \\
\hline AS 3610 IPRO & $0,11 \mathrm{c}^{(7)}$ & $0,59 \mathrm{~d}$ & 208889 & 115 & $16,99 \mathrm{e}$ & $3507 \mathrm{c}$ \\
\hline AS 3590 IPRO & $0,12 \mathrm{c}$ & $0,64 \mathrm{c}$ & 256667 & 130 & $19,38 \mathrm{~d}$ & 4819 a \\
\hline AS 3680 IPRO & $0,08 \mathrm{~d}$ & $0,67 \mathrm{c}$ & 200000 & 113 & $17,00 \mathrm{e}$ & $4106 \mathrm{~b}$ \\
\hline Caraíba Speed & $0,09 \mathrm{~d}$ & $0,60 \mathrm{~d}$ & 243333 & 114 & $15,61 \mathrm{f}$ & $3107 \mathrm{~d}$ \\
\hline Caraíba Nitro & $0,09 \mathrm{~d}$ & $0,56 \mathrm{~d}$ & 257778 & 114 & $16,98 \mathrm{e}$ & $3293 \mathrm{~d}$ \\
\hline 64i61RSF ${ }^{(1)}$ & $0,10 \mathrm{~d}$ & $0,69 \mathrm{c}$ & 221111 & 122 & $15,97 \mathrm{f}$ & 3964 c \\
\hline DM 66168 RSF IPRO & $0,10 \mathrm{~d}$ & $0,62 \mathrm{c}$ & 247778 & 133 & 22,18 a & 3893 c \\
\hline $68 \mathrm{i} 70 \operatorname{RSF}^{(2)}$ & $0,10 \mathrm{~d}$ & $0,58 \mathrm{~d}$ & 213333 & 127 & $20,63 \mathrm{~b}$ & $3587 \mathrm{c}$ \\
\hline $68 \mathrm{i} 68 \mathrm{RSF}^{(3)}$ & $0,09 \mathrm{~d}$ & $0,64 \mathrm{c}$ & 238333 & 140 & $20,03 \mathrm{c}$ & 2617 e \\
\hline HO Cristalino IPRO & 0,18 a & 0,89 a & 214444 & 140 & $15,63 \mathrm{f}$ & 5332 a \\
\hline HO Maracaí IPRO & 0,17 a & $0,73 \mathrm{~b}$ & 228333 & 130 & $15,98 \mathrm{f}$ & $4487 \mathrm{~b}$ \\
\hline HO Iguaçu IPRO & $0,10 \mathrm{~d}$ & $0,66 \mathrm{c}$ & 269167 & 118 & $13,74 \mathrm{~g}$ & $3331 \mathrm{~d}$ \\
\hline LG 60162 IPRO & $0,09 \mathrm{~d}$ & $0,55 \mathrm{~d}$ & 228333 & 114 & $15,97 \mathrm{f}$ & $3382 \mathrm{~d}$ \\
\hline LG 60174 IPRO & $0,10 \mathrm{~d}$ & $0,65 \mathrm{c}$ & 264167 & 130 & $15,71 \mathrm{f}$ & $4286 \mathrm{~b}$ \\
\hline LG 60179 IPRO & $0,13 \mathrm{c}$ & 0,85 a & 208889 & 137 & $13,24 \mathrm{~g}$ & 4791 a \\
\hline NS 6906 IPRO & $0,10 \mathrm{~d}$ & $0,66 \mathrm{c}$ & 226667 & 123 & $20,40 \quad b$ & 3496 c \\
\hline NS 7709 IPRO & $0,11 \mathrm{c}$ & $0,66 \mathrm{c}$ & 201667 & 130 & 19,98 c & 3969 c \\
\hline NS 6700 IPRO & $0,14 \mathrm{~b}$ & $0,73 \mathrm{~b}$ & 222222 & 137 & $19,50 \mathrm{c}$ & $4480 \mathrm{~b}$ \\
\hline TMG 7067 IPRO & $0,11 \mathrm{c}$ & $0,62 \mathrm{c}$ & 201667 & 125 & $19,22 \mathrm{~d}$ & $4395 \mathrm{~b}$ \\
\hline $1 \mathrm{XT}-\mathrm{I}$ & $0,12 \mathrm{c}$ & $0,62 \mathrm{c}$ & 205000 & 118 & 19,99 c & $3686 \mathrm{c}$ \\
\hline $2 \mathrm{XN}$ & $0,10 \mathrm{~d}$ & $0,64 \mathrm{c}$ & 248889 & 130 & $18,57 \mathrm{~d}$ & $3121 \mathrm{~d}$ \\
\hline 3 YM-I & $0,11 \mathrm{~d}$ & 0,50 e & 191667 & 120 & $18,99 \mathrm{~d}$ & $3644 \mathrm{c}$ \\
\hline 4 YB-I & $0,10 \mathrm{~d}$ & $0,62 \mathrm{c}$ & 241111 & 118 & $16,58 \mathrm{e}$ & $3833 \mathrm{c}$ \\
\hline $6 \mathrm{WB}-\mathrm{I}$ & $0,13 \mathrm{c}$ & $0,59 \mathrm{~d}$ & 240000 & 114 & $18,85 \mathrm{~d}$ & $4020 \mathrm{c}$ \\
\hline $\mathrm{CV}^{(5)}(\%)$ & 15,52 & 5,11 & & & 3,75 & 9,80 \\
\hline
\end{tabular}

\footnotetext{
${ }^{(1)}$ Brasmax Fibra IPRO; ${ }^{(2)}$ Brasmax Ícone IPRO; ${ }^{(3)}$ Brasmax Única IPRO; ${ }^{(4)}$ Brasmax Foco IPRO;

Coeficiente de variação; ${ }^{(6)}$ Altura de inserção: altura de inserção da primeira espiga; ${ }^{(7)}$ significativo a $5 \%$ de probabilidade.
}

A produtividade média de grãos, nos oito locais, variou de 2172 a $3471 \mathrm{~kg}^{\mathrm{ha}}{ }^{-1}$, inferior às produtividades constatadas por Borges et al. (2018) na safra 2017/18, que verificaram produtividades variando de 3295 a $4331 \mathrm{~kg} \mathrm{ha}^{-1}$, em Guararapes (cidade vizinha à Araçatuba), Manduri, Pindorama e Riolândia. Segundo Evans (1993), o potencial de produtividade de grãos pode ser definido como a produção de uma cultivar no ambiente ao qual está adaptada, sem limitações edafoclimáticas e nutricionais, livre da ação de pragas e doenças e com os demais estresses efetivamente controlados. 
Enfatiza-se que as condições onde foram realizados os experimentos devem ser consideradas, pois, a produtividade da cultura é definida pela interação entre planta, ambiente de produção e manejo (MAUAD et al., 2010) e, para uma planta manifestar o seu máximo potencial genético, caracterizado pelo seu melhor crescimento e desenvolvimento, diversos fatores ambientais podem influenciar diretamente no processo, como fotoperíodo, temperatura, radiação solar, nutrientes e vento (YUYAMA, 1991) e, segundo Sediyama (1989), fatores como temperatura, umidade, fertilidade do solo, época de semeadura e densidade de plantas afetam a altura de plantas, o grau de acamamento e a produtividade de grãos.

\section{CONCLUSÃO}

As cultivares avaliadas apresentaram, em média, massa de cem grãos variando de 12,37 a 17,19 g e produtividade de grãos variando de 2172 a $3471 \mathrm{~kg} \mathrm{ha}^{-1}$, nos oito locais avaliados no Estado de São Paulo.

\section{AGRADECIMENTOS}

Aos produtores Alan Fadil e Vanildo Florian Naressi que cederam as áreas para o desenvolvimento dos experimentos.

A todos os funcionários e estagiários da APTA e da CDRS, pelo apoio na condução dos experimentos.

Às empresas Caraíba Sementes LTDA; GDM Genética do Brasil S/A; Seedcorp HO Produção e Comercialização de Sementes S/A; Bela Sementes; Agroeste Sementes, Limagrain - LG Sementes; Agroata - Agropecuária Araçatuba; Cimoagro - Comércio e Representação Agropecuária LTDA, pelo apoio ao projeto que originou este trabalho.

\section{REFERÊNCIAS}

BERNARD, R. L.; CHAMBERLAIN, D.W.; LAWRENCE, R. D. Results of the cooperative uniform soybeans tests. Washington: USDA, 1965. $134 \mathrm{p}$.

BORGES, W. L. B.et al. Soybean cultivars regional evaluation in São Paulo State, Brazil - Season 2017/18. Nucleus, v. 1, p. 73-89, 2018. (Edição especial). Disponível em: http://www.nucleus.feituverava.com.br/index.php/nucleus/article/view/3006/2652

CIIAGRO - CENTRO INTEGRADO DE INFORMAÇÕES AGROMETEOROLÓGICAS . Resenha agrometeorológica, no período de 01/11/2018 a 01/04/2019. São Paulo, 2019. Disponível em: http://www.ciiagro.sp.gov.br/ciiagroonline/

CONAB - COMPANHIA NACIONAL DE ABASTECIMENTO. Acompanhamento da Safra Brasileira de Grãos. v. 6 - Safra 2018-19, n. 8 - Oitavo levantamento. Maio 2019. Disponível em: https://www.conab.gov.br/info-agro/safras/graos

CRUZ, T. V.et al. Componentes de produção de soja em diferentes épocas de semeadura, no oeste da Bahia. Bioscience Journal, v. 26, n. 5, p. 709-716, 2010. Disponível em: http://www.seer.ufu.br/index.php/biosciencejournal/article/viewFile/7198/5259 
DAY, P. R. Particle fractionation and particle-size analysis. In: BLAKE, C. A.et al. Methods of soil analysis: physical and mineralogical properties, including statistics of measurement and sampling. Madison: American Society of Agronomy, 1965. p. 545-567. (Part 1)

EVANS, L. T. Crop evolution, adaptation and yield. Cambridge University, Cambridge, 1993. 500 p. https://pubag.nal.usda.gov/pubag/downloadPDF.xhtml?id=11615\&content=PDF

FUNDAÇÃO MT. Boletim de Pesquisa de Soja. Rondonópolis: Fundação de Apoio a Pesquisa Agropecuária de Mato Grosso - Fundação MT, 2007. P. 63-128. (Boletim de Pesquisa de Soja, n. 11)

KOMORI, E.et al. Influência da época de semeadura e população de plantas sobre as características agronômicas da cultura da soja. Bioscience Journal, v. 20, n. 3, p. 13-p14, 2004. Disponível em: http://www.seer.ufu.br/index.php/biosciencejournal/article/view/6536/4270

MAUAD, M.et al. Influência da densidade de semeadura sobre características agronômicas na cultura da soja. Agrarian, v. 3, n. 9, p. 175-181, 2010. Disponível em:

http://ojs.ufgd.edu.br/index.php/agrarian/article/view/75

PEIXOTO, C. P.et al. Épocas de semeadura e densidades de plantas de soja: I. Componentes da produção e rendimento de grãos. Scientia Agrícola, v. 57, n. 1, p. 89-96, 2000. Disponível em: http://dx.doi.org/10.1590/S0103-90162000000100015

RAIJ, B. van et al.Análise química para avaliação da fertilidade do solo. Campinas: Instituto Agronômico; 2001.

SEDIYAMA, T.et alL. Cultura da soja: $1^{\text {a }}$ parte. Viçosa: UFV, 1989.

SILVA, F. A. S.; AZEVEDO, C. A. V. The Assistat Software Version 7.7 and its use in the analysis of experimental data. African Journal of Agricultural Research, v. 11, n. 39, p. 3733-3740, 2016. Disponível em:

http://www.academicjournals.org/journal/AJAR/article-abstract/5E8596460818

VERNETTI, F. J. Soja: planta, clima, pragas, moléstias e invasoras. Campinas: Fundação Cargil, 1983. v. 1.

YUYAMA, K. Avaliação de algumas características agronômicas e morfofisiológicas de cinco cultivares de soja (Glycine max (L.) Merril), cultivados em solo de várzea e de terra firme da Amazônia Central. 1991. 123 f. Tese (Doutorado) - Universidade Estadual Paulista, Jaboticabal, 1991. 